\title{
The Block Retrieval Problem
}

\author{
Marcos de Melo da Silva ${ }^{\mathrm{a}}$, Güneş Erdoğan ${ }^{\mathrm{b}, *}$, Maria Battarra ${ }^{\mathrm{b}}$, Vitaly Strusevich ${ }^{\mathrm{c}}$ \\ ${ }^{a} \operatorname{LIPN}$ (UMR CNRS 7030) - Institut Galilée - Université Paris-Nord, 99, \\ avenue Jean-Baptiste Clément, 93430 Villetaneuse, France. \\ ${ }^{b}$ School of Management, University of Bath, BA1 7AY, UK. \\ ${ }^{c}$ Department of Mathematical Sciences - University of Greenwich, Park Row, \\ London SE10 9LS, United Kingdom.
}

\begin{abstract}
Retrieving containers from a bay in a port terminal yard is a time consuming activity. The Block Retrieval Problem (BRTP) aims to minimize the number of relocations, the unproductive moves of hindering containers, while retrieving target containers belonging to a customer. The choice of relocations leads to alternative bay configurations, some of which would minimize the relocations of forthcoming retrievals. The Bi-objective Block Retrieval Problem (2BRTP) includes a secondary objective, the minimization of the expected number of relocations for retrieving the containers of the next customer. This paper provides $\mathcal{N} \mathcal{P}$-Hardness proofs for both the BRTP and 2BRTP. A branchand-bound algorithm and a linear time heuristic are developed for the BRTP; a branch-and-bound algorithm and a beam search algorithm are presented for the 2BRTP. Extensive computational tests on randomly generated instances as well as instances adapted from the literature are performed, and the results are presented.
\end{abstract}

Keywords: combinatorial optimization, container terminals, import containers, branch-and-bound

\section{Introduction}

Container terminals are exchange hubs for containers flowing from one transportation mode to another, or between container ships. Container terminals are typically located at ports, where containers are loaded to/unloaded from cargo boats and delivered to the customers for the last mile transportation. Containers can be classified as export (or outbound) containers, import or (inbound) containers, and transshipment containers (Kim and Park 2003, Caserta et al. 2011). Export containers arrive by trucks or trains to the terminal landside area, then they are placed in the storage yard by internal vehicles, and relocated to the seaside area when the corresponding cargo boat is available (Daganzo 1989). Import containers follow the reverse path; they arrive in the port by cargo boats, then they are unloaded by quay cranes, and placed in the storage yard to be picked up by trucks or trains in the landside area. Transshipment containers are restricted to the seaside and the storage yard areas; they are unloaded from a vessel and stored in the port yard until they are loaded to another cargo boat. A schematic representation of a container terminal together with a classification of container flows is depicted in Figure 1.

Container terminals provide temporary storage space for preventing the need of synchronization between the transportation modes, in addition to their function as exchange areas. Thus, containers arriving in the terminal by ship, or by external trucks, or by trains are temporarily kept in storage areas until they are requested for shipment. The dwell times for export, import, and transshipment containers are different. Export containers typically arrive in the port up to weeks before the time

\footnotetext{
${ }^{*}$ Corresponding author

Email addresses: marcos.demelodasilva@lipn.univ-paris13.fr (Marcos de Melo da Silva), G.Erdogan@bath.ac.uk (Güneş Erdoğan), M.Battarra@bath.ac.uk (Maria Battarra), V.Strusevich@greenwich.ac.uk (Vitaly Strusevich)
} 


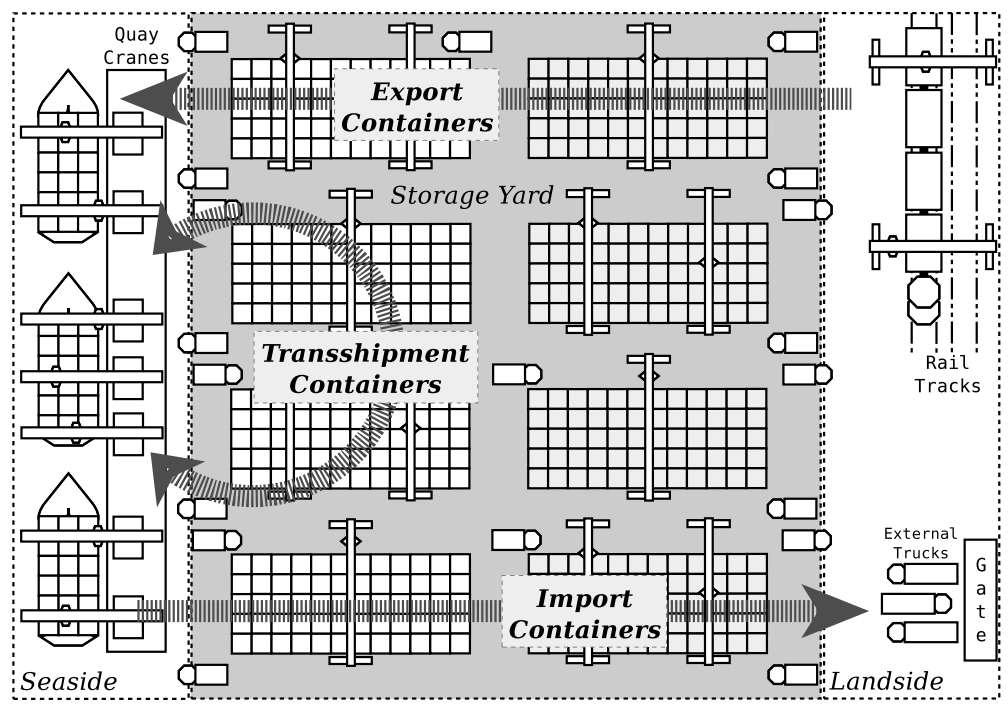

Figure 1: A typical container terminal and the three types of container flows.

they have to be shipped and import containers stay in the terminal yard until they are claimed by the customers.

The storage yard is a scarce resource just as berths, cranes, and internal vehicles, and its usage needs to be carefully planned. Containers are stored in stacks in order to avoid spreading them around the terminal yard, which would have required larger areas and would have demanded a more substantial transportation effort. Stacks are aligned to form bays and blocks, as illustrated in Figure 2. This configuration optimizes the space utilization and allows for crane operations. Nevertheless, by adopting this storage policy, a trade-off between space saving and handling effort for loading and unloading operations arises. More precisely, hindering or obstructing containers may need to be relocated in order to provide access to the blocked target containers during a retrieval request.

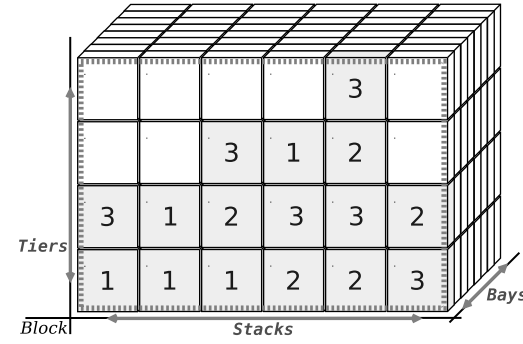

(a)

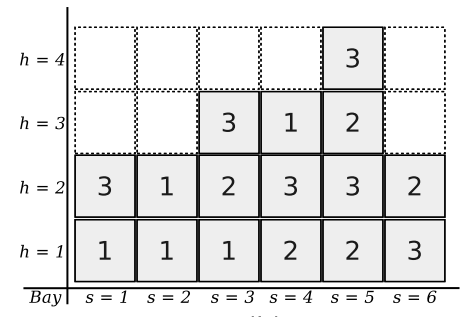

(b)

Figure 2: (a) Container storage block, and corresponding (b) bay representation.

We assume each bay stores uniformly shaped containers, consisting of $S$ stacks of maximum height $H$. Each container belongs to a group $g \in\{1, \ldots, G\}$ which can be defined according to its weight, destination, owner, or any other classification criterion that can be used during the retrieval process. Containers in a group have to be retrieved at the same time. The target group refers to containers of the group $t \in\{1, \ldots, G\}$ which are about to be retrieved (note that $t>1$ is allowed, in contrast to the related problems in the literature). The LIFO policy has to be observed; i.e., only the topmost containers can be directly reached by a crane or a gantry. Thus, retrieving a container that is not positioned in the top tier requires all containers above it to be moved to other stacks. Obstructing containers on top of target containers are called deadlocks. A relocation occurs when a 
deadlock has to be moved from one stack to another. A retrieval is performed when a container of the target group is picked and leaves the bay. It is assumed that containers can be relocated to other stacks and hence there exists enough space above the stacks to perform the necessary relocations. In practice, it is not always possible to assure enough space above the stacks to perform the necessary relocations, in such cases the non-target containers are relocated to a temporary dummy stack at the side of the bay. Once the retrieval process is finished, the containers in the dummy stack have to be put back into the bay.

Although it is optimal to place the containers of a group in adjacent empty stacks and bays upon arrival, lack of available space may force the group to be divided in partially occupied stacks and non-adjacent locations. Furthermore, retrieval operations for the other groups may further disperse the containers within the same bay and cause deadlocks. In the Block Retrieval Problem (BRTP), the aim is to retrieve all containers of a given target group $t, 1 \leq t \leq G$, provided that the relocation cost $C_{t}$ is minimized. In this paper, the cost $C_{t}$ is defined as the number of relocations needed to retrieve all the containers of the target group $t$. Even if there are several groups other than the target group $t$, in the BRTP there is no distinction between them, i.e., essentially there are only two groups: the given target group $t$ and the other group formed by all remaining containers.

The BRTP is important in its own right; however, its objective is myopic, since it does not consider a configuration of the deadlocks once they have been relocated in the bay, although that could be important for retrievals of the forthcoming target groups. The Bi-objective Block Retrieval Problem (2BRTP) overcomes this limitation. The 2BRTP is a bi-criteria problem with two lexicographically ordered objectives: the primary objective is to minimize the $\operatorname{cost} C_{t}$ of the retrieval of the initial target group $t$, and the secondary objective is to minimize the expected number of relocations of the forthcoming retrieval. The lexicographic objective function reflects the priority given to the customer currently at the port for the retrieval. However, among the fastest retrieval solutions for the current customer, the solution resulting in the most promising bay configuration for the upcoming customers is chosen by means of the secondary objective function. Assuming that the probability that a group $g, 1 \leq g \leq G, g \neq t$, is retrieved after the first target group $t$ is known and is equal to $P_{g}$, the secondary objective function can be written as $\sum_{g \in\{1, \ldots, G\} \backslash t} P_{g} C_{g}$, where $C_{g}$ is the cost of the retrieval of group $g$ from the bay of the configuration obtained after the containers of the primary target group $t$ have been retrieved at the minimum cost $C_{t}$. Figure 3a illustrates an instance of the 2BRTP. In both solutions shown in Figures $3 \mathrm{~b}$ and $3 \mathrm{c}$ the smallest relocation cost is $C_{1}=12$, i.e., each of these solutions can be considered as an optimal solution to the BRTP. However, for the configuration shown in Figure 3b the secondary objective used for the 2BRTP is $2 \times P_{2}$, while in the configuration in Figure $3 \mathrm{c}$ there are no deadlocks, so that it corresponds to an optimal solution of the 2BRTP.

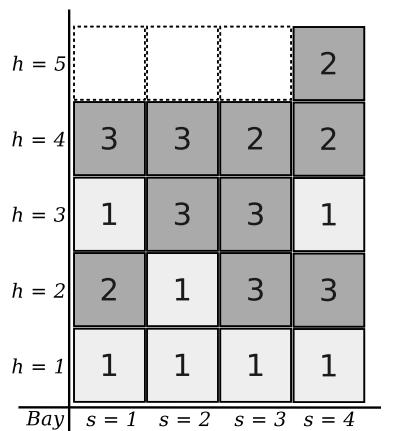

(a)

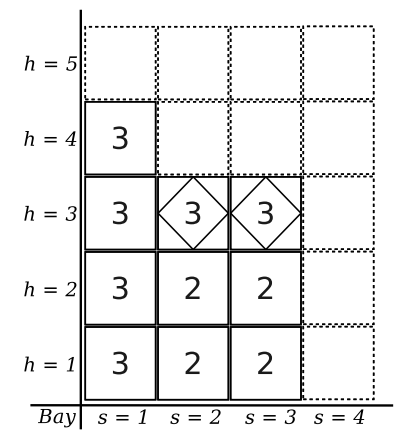

(b)

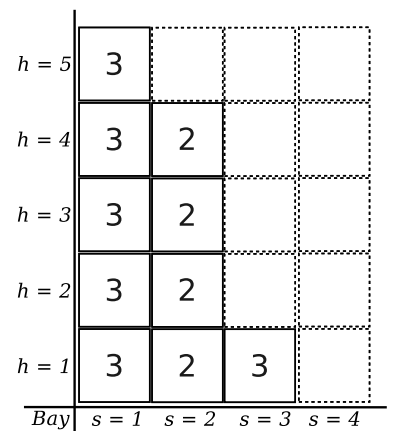

(c)

Figure 3: (a) A bay with 4 stacks of maximum height 5 holding containers partitioned into 3 groups, target group 1 and obstructing containers in dark gray, (b) BRTP solution where the future deadlocks are diamond shape, (c) 2BRTP optimal solution. 
The remainder of this paper is organized as follows. Section 2 provides a literature review. We present a proof of $\mathcal{N} \mathcal{P}$-Hardness for the BRTP (Section 3.1) and we show that the BRTP can be solved in polynomial time if $S$ is constant (Section 3.2). A B\&B algorithm for the BRTP (Section 3.3) and a linear algorithm for solving a special class of BRTP instances (Section 3.4) are provided. We present a proof of $\mathcal{N} \mathcal{P}$-Hardness for the 2BRTP (Section 4.1) as well as a B\&B algorithm (Section 4.2), and a beam search heuristic (Section 4.3). Extensive computational experiments have been performed on newly generated instances and adaptations of instances from the literature, the results of which are in Section 5. Finally, Section 6 provides our concluding remarks.

\section{Literature review and motivation}

In this section, we briefly review known results on various block retrieval models, keeping focus on the differences between these models and the BRTP and the 2BRTP. We also give motivational reasons for studying the BRTP and the $2 \mathrm{BRTP}$, from both practical and theoretical points of view.

The turnaround time of vessels, trains and external trucks are key factors to measure the efficiency of container terminals, and also contribute to the evaluation of customer service levels and port competitiveness (Kim and Kim 1999). We refer the interested reader to the recent paper by Lee and Song (2017) for a critical overview of the literature on container transport in global supply chains. Among many factors that may affect the overall turnaround time, loading and unloading operations are the most time consuming tasks performed in terminal yards, and therefore they need to be optimized (Roberti and Pacino 2016). The BRTP and 2BRTP aim at improving the retrieval times, in particular in the landside area of the port terminal. The amount of retrieval information differs for import and export containers (Caserta et al. 2011). The loading sequence on a cargo boat for export containers is typically known when a cargo boat approaches the berth, whereas the retrieval sequence for import containers is typically revealed during the delivery process. Trucks or trains may arrive at the container terminal for the retrieval at unspecified times. The landside retrieval process has been less investigated in the literature. In the following, we provide a summary of related problems.

A recent survey and classification scheme for loading, unloading, and pre-marshalling operations in stack storage contexts is proposed by Lehnfeld and Knust (2014). For additional contributions and surveys on container terminals and their operations see Steenken et al. (2004), Dekker et al. (2006), Vis and Roodbergen (2009), Carlo et al. (2013, 2014a,b), Gharehgozli et al. (2015), Ku and Arthanari (2016), Liu et al. (2016).

A popular stacking model that arises in storage yards is the Block Relocation Problem (BRP). In this problem, it is required to minimize total relocation cost, provided that all groups have to be retrieved in a known sequence (Kim and Hong 2006, Caserta and Voß 2009, Tanaka and Takii 2016). Thus, the BRTP is a special case of the BRP where there are only two groups, with the target group to be retrieved first, and the rest of the containers next.

There are several issues that motivate our study of the BRTP. First, the computational complexity of this simplest version of the BRP has to be established, and this is done in Sections 3.1 and 3.2. Second, mathematical formulations for the BRP presented in Caserta et al. (2012) and Zehendner et al. (2015) show that the exact algorithms for the problem are limited to relatively small sizes. Although the BRTP can be solved by the available algorithms developed for a more general BRP, it is reasonable to expect that the algorithms tailored for solving the BRTP that explore its simpler structure would performer better than general algorithms. We offer a simple linear-time heuristic for solving the BRTP, provided that the bay has enough room for moving the deadlocks. Besides, we have designed a branch-and-bound (B\&B) algorithm for the BRTP; see Section 3.3 and Algorithm 1. We have also performed computational experiments comparing our B\&B algorithm with the B\&B algorithm by Tanaka and Takii (2016) for the BRP. Up to this date, the latter algorithm remains the fastest exact algorithm for the BRP that can deal with grouped instances. We compare the two algorithms on randomly generated BRTP instances with $S=10$ stacks, maximum height up to $H=15, G=\{2,3\}$, and filling rate of $95 \%$; in total 4000 instances were randomly generated 
as described in Section 5.1. In our experiments we employed the original code made available by Tanaka and Takii (2016). We have observed that our B\&B algorithm for the BRTP is capable of solving more instances to optimality in less time than the BRP B\&B algorithm from the literature. The difference in performance is negligible for smaller instances. These results are summarized in Table 12 of Appendix C.

It is clear that the BRTP being the simplest block retrieval problem may have a rather limited practical usage. To a very large extend, our study on the BRTP is a starting point that allows us to define the 2BRTP, which we see as the main contribution of this paper. Still, the results obtained for the BRTP are of a certain interest in their own right, and the designed algorithms serve as building blocks for the exact and heuristic algorithms for the 2BRTP.

The 2BRTP assumes that the first target group is known, but the forthcoming group to be retrieved is uncertain. Thus, the $2 \mathrm{BRTP}$ cannot be modeled as a special case of the BRP. Moreover, examples of small-sized instances can be provided that demonstrate that the 2BRTP does not reduce to a series of the BRP with various retrieval sequences. This makes $2 \mathrm{BRTP}$ an object on independent mathematical study. From the application point of view, both the BRTP and the 2BRTP can be utilized for optimized collection of containers of a single customer dispersed to multiple bays, by solving the associated problem for each of these bays. In terms of the container flow in seaports, the BRP better models the retrieval of export containers to be loaded into vessels (in which the loading sequence is deterministic), while the BRTP and 2BRTP suit the retrieval operations of import containers to be delivered to the landside transportation operator (in which the customers' arrival times are not known or are uncertain). In fact, out interest in studying the 2BRTP has arisen from the discussions of the second author with the field managers of a major container port in Istanbul, Turkey. Typically, the ports allow import containers to be stored without charging a storage fee for up to seven days, depending on the country and port. After that a so-called demurrage fee is applied. In this context, the term "demurage" refers to money that must be paid when goods are collected later than the agreed time after being taken off a chartered ship. The free time before demurrage allows the recipients to schedule the pickup to their convenience, and introduces uncertainty to the order or the retrieval operations.

The number of relocations needed to retrieve target containers from stacks is affected by the height and the width of the stacks and the strategy employed during the storage process. Castilho and Daganzo (1993) analyze two strategies which involve segregation and non-segregation of new containers arriving to the storage area (i.e., new containers are put in a dedicated area or they are mixed with the previously stored containers). They present methods for estimating the expected number of relocations when retrieving a single container and also when retrieving several containers from a group of stacks in two scenarios, with and without new containers being added to the stacks. Using simulation, the authors observe that in the scenario in which new containers enter the bay, the segregation strategy is advantageous if the throughput of incoming ships is small and the stacks are high. In the case of large ships throughput and short stacks, the non-segregating strategy results in a better performance.

Considering a given bay setting in which no incoming containers are allowed, Kim (1997) proposes a numerical approximation to estimate the expected number of relocations to retrieve an arbitrary container and also to retrieve all containers from the bay. The estimation approach outperforms other methods available (Watanabe 1991) in both accuracy and unbiasedness of the estimations.

The problem addressed in this paper differs from those described in the papers reviewed above. The contributions in the literature focus on the rehandling of import containers at the strategic level; i.e., searching for the best stacking dimensions and storage strategies for reducing or avoiding relocations during the retrieving process. This paper deals with the problem at an operational level, and provides fast algorithms for the retrieval of target containers.

Similarly, Borjian et al. (2015a) and Borjian et al. (2015b) study the Container (Blocks) Relocation Problem and the Blocks Relocation Problem with Incomplete Information. For the latter, the authors assume that the retrieval sequence of some container groups is known, while for the remaining groups only a probability distribution of the retrieval order is given. In addition, the true 
retrieval sequence of the unknown groups is revealed during the execution of the algorithm. For the BRP, they introduce service time windows among other side constraints, and provide an integer programming formulation. For the BRP with Incomplete Information, the authors adopt an $A^{*}$ algorithm from the literature and combine it with a sampling technique.

\section{The Block Retrieval Problem}

In this section, we prove that the BRTP is $\mathcal{N} \mathcal{P}$-Hard if the number of stacks is variable and admits a polynomial-time solution algorithm for a fixed number of stacks. We also describe a branch-and bound (B\&B) algorithm and a linear time algorithm for a restricted set of BRTP instances. These algorithms are then used as building blocks for developing algorithms for 2BRTP in Section 4.

An instance of the BRTP can be described by providing the following information. There are $S \geq 2$ stacks such that stack $i$ contains $h_{i}$ tiers numbered from bottom to top, i.e., may contain up to $h_{i}$ containers. Let us call $h_{i}$ the height of stack $i$ and define $H=\max \left\{h_{i} \mid 1 \leq i \leq S\right\}$, the maximum height. All these values are initial characteristics of the bay and remain constant. We distinguish between the target containers and non-target containers. Considering a stack $i$ in the order of numbering of its tiers, let $u_{i, 0}$ be the number of non-target containers at the bottom of the stack; if there are no such containers then $u_{i, 0}=0$. The remaining containers are organized in $p_{i}$ pairs of the form $\left(w_{i, j}, u_{i, j}\right)$, where for the $j$-th pair, $1 \leq j \leq p_{i}, w_{i, j}$ is the number of contiguous slots with the target containers while $u_{i, j}$ is the number of contiguous slots with the non-target containers. Thus, a configuration of stack $i$ can be described by a string of the form

$$
Q_{i}=\left[u_{i, 0},\left(w_{i, 1}, u_{i, 1}\right), \ldots,\left(w_{i, p_{i}}, u_{p_{i}}\right)\right] .
$$

Visual representation of a stack defined by the string $Q_{i}=[0,(1, X),(1,1)]$ is depicted in the leftmost column of the bay in Figure 4a. The objective of BRTP is to retrieve all target containers making the smallest possible number of relocations of non-target containers.

\subsection{BRTP with Variable Number of Stacks}

We prove the $\mathcal{N} \mathcal{P}$-Hardness of the BRTP by providing a valid reduction from the well-known $\mathcal{N} \mathcal{P}$-Complete problem Partition.

Recall the definition of PARTiTion from Garey and Johnson (1979). Given positive integers $a_{1}, \ldots, a_{n}$ and the index set $N=\{1, \ldots, n\}$ such that $\sum_{i \in N} a_{i}=2 A$, is it possible to partition set $N$ into disjoint subsets $N_{1}$ and $N_{2}$ such that $\sum_{i \in N_{1}} a_{i}=\sum_{i \in N_{2}} a_{i}=A$ ?

Proposition 3.1. The Block Retrieval Problem (BRTP) is $\mathcal{N} \mathcal{P}$-Hard in the ordinary sense, provided that the number of stacks $S$ is variable.

Proof Proof. Given an instance of PARTition, denote

$$
\begin{aligned}
\hat{a} & =\max \left\{a_{i} \mid i \in N\right\}, \\
X & =2 n \hat{a}-3 A .
\end{aligned}
$$

Define the following instance of the BRTP. The number of stacks is $S=n+1$ and the height of each of them is $H=2 \hat{a}+X+1$. Each stack $i, 1 \leq i \leq n$, is described by the configuration

$$
Q_{i}=\left[0,(1, X),\left(a_{i}, a_{i}\right)\right] .
$$

Thus, starting from the bottom of stack $i$, its content is a single target container, a set of $X$ non-target containers, $a_{i}$ target containers and $a_{i}$ non-target containers. The configuration of stack $n+1$ is given by

$$
Q_{n+1}=[0,(1, X)]
$$

We show that for the created instance the cost of retrieving all target containers is at most $(n+2) X+3 A$ if and only if PARTITION has a solution. Note that the total number of the deadlocks 
in this instance is $(n+1) X+2 A$. For this instance, the length of input of PARTition is bounded above by $n \log \hat{a}$. To compute $\hat{a}$ and $X$, we need $\mathcal{O}(n)$ operations, and the content of each stack requires at most $\log X+2 \log \hat{a}$ bits, so that the described reduction is polynomial with respect to the length of input of PARTITION.

For an instance of PARTITION with $n=4$ and $a_{1}=1, a_{2}=a_{3}=2$ and $a_{4}=3$, the structure of the corresponding instance of the BRTP is shown in Figure 4a. The target containers are labeled by "1" while the non-target containers are labelled by "0" $\dot{W}$ e will use this example to illustrate parts of the proof.

Necessity $(\Rightarrow)$ : Assume that Partition has a solution, so that $N_{1}$ and $N_{2}$ are the required subsets such that $N_{1}=\left\{i_{1}, \ldots, i_{q}\right\}$. Notice that the total number of free slots in all stacks $i$, $1 \leq i \leq n+1$, is equal to $(n+1)(H-X-1)-4 A=2(n+1) \hat{a}-4 A=X-A+2 \hat{a}$, including $2 \hat{a}$ free slots in stack $n+1$. Perform the following actions:

1. For stack $i_{1}$, move $a_{i_{1}}$ non-target containers to stack $n+1$ and retrieve $a_{i_{1}}$ target containers.

2. For each $k$ from 2 to $q$, for stack $i_{k}$, move $a_{i_{k}}$ non-target containers to stack $i_{k-1}$ and retrieve $a_{i_{k}}$ target containers.

This transformation requires $\sum_{k=1}^{q} a_{i_{k}}=A$ moves and creates $A$ additional free slots. As a result of this transformation, there exists at least one stack $i_{q}$ containing one target container at the bottom and $X$ non-target on top, i.e., its current configuration is $Q_{i_{q}}=[0,(1, X)]$, and the total number of free slots in all other stacks is equal to $X$. This bay configuration is illustrated in Figure $4 \mathrm{~b}$, where we have taken $N_{1}=\{1,4\}$, i.e., $a_{1}=1$ container is moved from stack 1 to stack 5 , followed by the relocation of $a_{4}=3$ containers from stack 4 to stack 1 .

After actions 1 and 2 are completed, we may proceed as follows.

3. Move $X$ non-target containers from stack $i_{q}$ to stacks with available space, make stack $i_{q}$ empty by retrieving the bottom target container (see Figure 4c).

4. Process non-empty stacks with target containers in any order. Empty each stack by moving the non-target containers into an empty stack and retrieving all target containers.

After Step 3, stack $i_{q}$ is empty, with $H$ free slots, while all other $n$ stacks are completely full. In each iteration of Step 4, a stack with target containers is made empty, and its non-target containers are moved to the stack that is empty in the beginning of the iteration. In Step 4, each of the $(n+1) X+2 A$ non-target containers is relocated exactly once. In the end of this process, all target containers will be retrieved. Together with $A+X$ moves performed in Steps 1, 2 and 3, the total number of moves is $(n+2) X+3 A$.

Sufficiency $(\Leftarrow)$ : Suppose that for the constructed instance of the BRTP, there exists a solution with at most $(n+2) X+3 A$ moves. We now demonstrate that no solution can be obtained with less than $(n+2) X+3 A$ relocations. During the relocation process, a situation arises that we call Event $R$ : some stack $k$ contains only one target container at the bottom and $X$ non-target containers on top, and there is enough room in all other stacks to relocate the $X$ non-target containers from stack $k$. Denote the number of moves that lead to Event $R$ by $T(R)$. If Event $R$ occurs, all target containers can be retrieved, starting from stack $k$, in $(n+2) X+2 A$ additional moves. If $T(R)>A$, then the total number of moves exceeds $(n+2) X+3 A$, which contradicts our supposition. Thus, $T(R) \leq A$.

Consider the process of reaching Event $R$. We cannot start with the relocation of the $X$ nontarget containers from stack $n+1$, since the number of the free slots in all other stacks is $X-A$. Thus, we will move non-target containers from the top pair of some stacks $i, 1 \leq i \leq n$. If Event $R$ is reached after $T(R)$ such moves are performed, then $T(R)$ target containers are retrieved, the total number of all free slots is $X-A+T(R)+2 \hat{a}$, and there exists a stack $k$ with only one target container at the bottom, $X$ non-target on top and $2 \hat{a}$ free slots in that stack. In the case of Event $R$, we must have at least $X$ free slots in all stacks other than $k$, which is only possible if $T(R)=A$. 


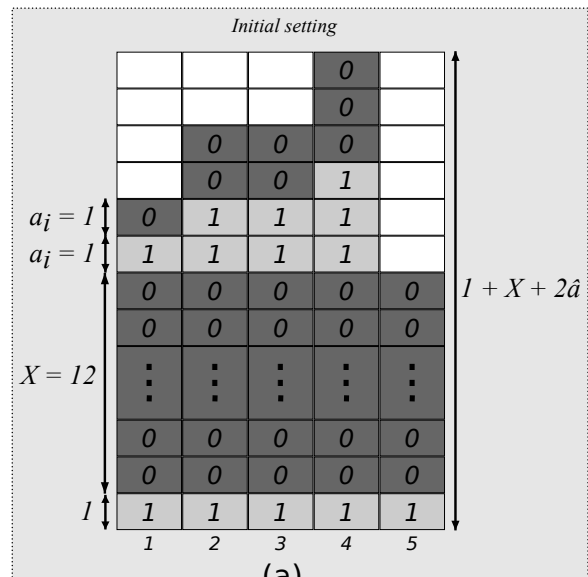

(a)

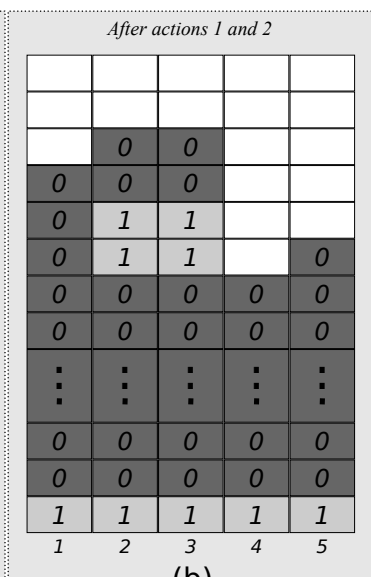

(b)

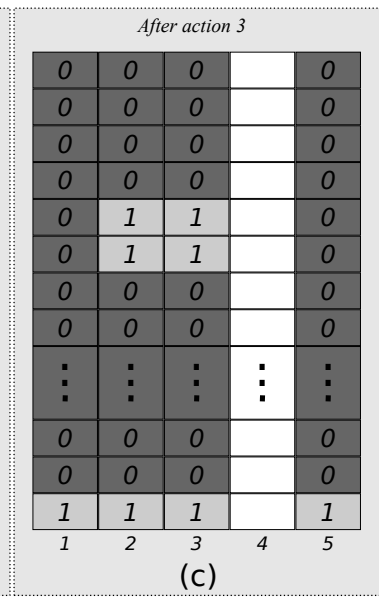

(c)

Figure 4: BRTP instance for PARTITION set $a=\{1,2,2,3\}$, and bay layouts after performing actions 1,2 , and 3 .

Denote by $N_{1}$ the set of stacks from which the target containers are retrieved in order to reach Event $R$, and define $N_{2}=N \backslash N_{1}$. These two sets form a solution to PARTition. Q.E.D.

\subsection{Fixed Number of Stacks}

In this subsection, we show that the BRTP is solvable in polynomial time with respect to the number of containers (or, equivalently, the maximum stack height $H$ ), provided that the number of stacks is fixed. We will employ a dynamic programming (DP) algorithm for this purpose.

Given an instance of the BRTP represented by $S$ strings $Q_{i}, 1 \leq i \leq S$, we redefine $h_{i}:=h_{i}-u_{i, 0}$, since the non-target containers below all target containers are not relocated and only affect the number of free slots in a stack.

As a preprocessing part of the algorithm, for each stack $i, 1 \leq i \leq S$, compute $f_{i, k}$, the number of slots above the $k$-th pair

$$
\begin{aligned}
& f_{i, 0}=h_{i}, \\
& f_{i, k}=f_{i, k-1}-\left(w_{i, k}+u_{i, k}\right), k=1, \ldots, p_{i} .
\end{aligned}
$$

We show that the problem with a fixed number of stacks can be solved by a DP algorithm. The algorithm uses states of the form:

$$
\left(k_{1}, x_{1} ; k_{2}, x_{2} ; \ldots ; k_{S}, x_{S}\right)
$$

where

$k_{i}$ is the number of pairs in stack $i$ in the current partial solution; if $k_{i}=0$ then stack $i$ may only contain non-target containers;

$x_{i}$ is the number of non-target containers in the top pair in stack $i$; if $k_{i}=0$ then $x_{i}$ is the number of all (non-target) containers in the stack;

For a given state, we denote by $\Phi$ the current number of relocations, i.e., the smallest number of moves of non-target containers needed to achieve that state. The DP algorithm starts with the initial state $\left(p_{1}, u_{1, p_{1}} ; p_{2}, u_{2, p_{2}} ; \cdots ; p_{S}, u_{S, p_{S}}\right)$ associated with $\Phi\left(p_{1}, u_{1, p_{1}} ; p_{2}, u_{2, p_{2}} ; \cdots ; p_{S}, u_{S, p_{S}}\right)=0$.

Given a state of the form $\left(k_{1}, x_{1} ; k_{2}, x_{2} ; \ldots ; k_{S}, x_{S}\right)$, the algorithm selects a stack $t$ from which $x_{t}$ non-target containers will be relocated. For the case of general $S$, we have to generate all options of redistributing the non-target containers from a chosen stack. 
To facilitate these options, recall that a composition of an integer $u$ made of $v$ summands is a sequence $\left(z_{1}, z_{2}, \ldots, z_{v}\right)$ of positive integers such that $u=z_{1}+z_{2}+\cdots+z_{v}$. According to Flajolet and Sedgewick (2009), the number of compositions of $u$ into at most $v$ positive summands (i.e., exactly $v$ non-negative summands) is

$$
C_{u}^{(\leq v)}=\left(\begin{array}{c}
u+v-1 \\
v-1
\end{array}\right)
$$

which can be estimated as $\mathcal{O}\left(u^{v-1}\right)$.

A typical recursion can be written as follows. For a state of the form $\left(k_{1}, x_{1} ; k_{2}, x_{2} ; \ldots ; k_{S}, x_{S}\right)$, select a stack $t$ with $k_{t} \geq 1$. For a generated composition $x_{t}=z_{1}+\cdots+z_{t-1}+z_{t+1}+\cdots+z_{S}$ into $S-1$ non-negative summands, define the state

$$
\left(k_{1}, x_{1}+z_{1} ; \ldots ; k_{t-1}, x_{t-1}+z_{t-1} ; k_{t}-1, u_{t, k_{t-1}} ; k_{t+1}, x_{t+1}+z_{t+1} ; \ldots ; k_{S}, x_{S}+z_{S}\right),
$$

which is only feasible if the inequality

$$
f_{i, k_{i}-1}+x_{i}+z_{i} \leq h_{i}
$$

holds for each $i, 1 \leq i \leq S, i \neq t$. Notice that for stack $t$ the number of pairs decreases by 1 since $u_{t, k_{t}}$ non-target containers are relocated and then $w_{t, k_{t}}$ target containers are retrieved.

The process is repeated until all states of the form $\left(0, x_{1} ; 0, x_{2} ; \cdots ; 0, x_{S}\right)$ are found. If during the described computation, a state with the same values of state variables has already been reached, we keep only one state associated with the smallest value of the function $\Phi$. The smallest value of the function $\Phi$ associated with the states of the form $\left(0, x_{1} ; 0, x_{2} ; \cdots ; 0, x_{S}\right)$ is the optimal number of relocations. The sequence of relocations that solves the problem can be found by backtracking.

For a chosen stack $t, 1 \leq t \leq S$, the overall number of ways to redistribute $x_{t}$ non-target containers from the its top pair does not exceed the number of compositions of $x_{t}$ into exactly $S-1$ non-negative summands. Thus, the total number of states generated this way from a given state $\left(k_{1}, x_{1} ; k_{2}, x_{2} ; \ldots ; k_{S}, x_{S}\right)$ for a chosen stack $t$ is $\mathcal{O}\left(x_{t}^{S-2}\right)$, which for all stacks yields $\mathcal{O}\left(H^{S-2}\right)$. The total number of generated states is $\mathcal{O}\left(\gamma^{S} H^{S}\right), \gamma=\max \left\{p_{i} \mid 1 \leq i \leq S\right\}$, which gives the overall time complexity of $\mathcal{O}\left(\gamma^{S} H^{2 S-2}\right)$. Since $\gamma \leq H$, we deduce that the described DP algorithm requires $\mathcal{O}\left(H^{3 S-2}\right)$ time.

\subsection{A Branch-and-Bound Algorithm for the BRTP}

In this section, we present a B\&B algorithm to solve exactly the BRTP. The pseudocode of our $\mathrm{B} \& \mathrm{~B}$ algorithm is provided in Algorithm 1 and Appendix A provides additional information concerning the data structures used in the pseudocode of this algorithm, as well as the rest of the algorithms in the article.

A preprocessing step is performed in the initial bay in order to retrieve all the unobstructed target containers (i.e., in the top tiers). A lower bound on the number of deadlocks for the BRTP is computed as follows. For each stack $i \in\{1, \ldots, S\}$, the lower bound $L B_{i}$ on the number of relocations needed to retrieve all target containers is $\sum_{k=1}^{p_{i}} u_{i, k}$, and the global bound is $L B=\sum_{i=1}^{S} L B_{i}$. In addition, an upper bound on the total number of relocations needed to retrieve all target containers can be set to a high enough value or computed using a linear time algorithm (Algorithm 2 to be presented in Section 3.4), when applicable.

The $\mathrm{B} \& \mathrm{~B}$ root node is initialized with the initial configuration of the bay, and the branching strategy consists of enumerating all possible retrieval operations starting from the given bay configuration (and corresponding relocations). Algorithm 1 explores the B\&B search tree in a depth-first fashion. At each node of the tree, if the number of relocations executed so far for retrieving the target block plus the lower bound in the number of relocations that will be necessary to retrieve the remaining target containers is greater than or equal the best BRTP upper bound, the node is fathomed (lines 2 to 9 ). If any target container sits in the bay and no fathoming condition is reached, 
new nodes are created from a B\&B node (lines 10 to 56) by selecting a stack $s \in\{1, \ldots, S\}$ and retrieving target containers which are no longer obstructed (line 11). If such a stack does not exist, a pickup stack containing at least one target container is selected (line 37), as well as a delivery stack, in which the non target container will be placed (line 44). We note that the B\&B algorithm considers moving one container at a time instead of handling one pair at a time. This is to avoid the complexity of determining how to distribute the obstructing containers in a pair to the stacks with empty space.

Two strategies are employed in order to reduce the number of choices of pickup and delivery stacks. The strategy for choosing the pickup stack consists in using the same stack from the previous node, provided that there are obstructing containers above the target (line 22). If such stack is not available (e.g., in the beginning of the search or after retrievals), a stack $s \in\{1, \ldots, S\}$ that contains at least one target container is selected. Likewise, the strategy for delivery stacks consists in first relocating to those stacks with no target containers. If no stack meets this condition, any other stack $s^{\prime} \in\{1, \ldots, S\}$ different from the pickup stack is selected (line 28). Once the pair of stacks is chosen, the topmost container from the pickup stack is relocated to the delivery stack. Note that both strategies would be suboptimal for the 2BRTP.

Finally, if the lower bound on the number of relocations is smaller than the current upper bound, and there is no target container left in the bay, then a new best known BRTP solution is found. The incumbent solution is updated and the node is fathomed.

\subsection{A Linear Time Algorithm for the BRTP with empty slots}

In this section, we describe a linear time algorithm (in terms of $n$, the number of containers) that can solve to optimality BRTP instances in which at any step of the algorithm it is always possible to reach the lowest level target container in at least one stack, i.e.

$$
\exists k \in\{1, \ldots, S\} \mid L B_{k} \leq \sum_{i=1, i \neq k}^{S}\left(h_{i}-\sum_{j=0}^{p_{i}}\left(w_{i, j}+u_{i, j}\right)\right)
$$

During our testing, all the instances adapted from the literature and randomly generated (with bay sizes similar to instances for related problems from the literature) observed to be feasible and solvable with the linear time algorithm. We artificially generated instances to test how often Condition 2 is violated and observed that only unlikely configurations may produce infeasibility, a mere $3.4 \%$ of the artificially hard instances. Details will be provided in Section 5 , but the linear algorithm proved to be of practical relevance according to our computational tests.

We now present this algorithm for the BRTP, the pseudocode of which is provided in Algorithm 2. The trivial retrievals are performed at first; i.e., the target containers available at the top of the stacks. Next, $L B_{i}$ is computed for each stack $i \in\{1, \ldots, S\}$ and the stacks that do not hold target containers are marked as priority stacks, in linear time. The remaining stacks containing target containers are sorted with respect to their $L B_{i}$ in non-decreasing order in $\mathcal{O}(S)$ (linear) time using the Counting Sort algorithm (Cormen et al. 2009). The following steps are repeated until the sorted list is empty. The first stack in the sequence is selected and all obstructing containers positioned over the target containers are relocated to stacks marked as priority target stacks if available, otherwise the containers are relocated to the next stacks in the sorted sequence. This can be done in $\mathcal{O}(h)$ complexity, at most $\mathcal{O}(S)$ times, which brings the overall complexity to $\mathcal{O}(n)$. After retrieving all the target containers, the selected stack is removed from the sorted sequence and included in the priority target list. A priority stack with minimum height is chosen for the relocation process. The priority target list is implemented as a linked list of linked lists. The main list is sorted in nondecreasing order according to the number of containers in each non-target stack. Each element in the list is a linked list that contains all the non-target stacks with the same amount of containers. Using this data structure, the complexity for inserting new elements is $\mathcal{O}(H)$ (as in lines 11 and 31), and retrieving the less occupied stack and updating the list can be performed in constant time (as in line 23). The complexity of Algorithm 2 is $\mathcal{O}(n)$. 
Notice that the described heuristic is only applicable if there is enough room in the bay, so that it is always possible to reach the lowest target container in at least one stack. On the other hand, there are instances of the BRTP, even with a small number of stacks, e.g., $S=2$, such that the problem either admits no solution or the running time for finding an optimal solution is not linear with respect to the maximum height $H$, or to the total number of containers $n$ in the original setup.

For an illustration, assume that $S=2$ and the height of each stack is even, i.e., $H=2 h$. The structure of Stack 1 is described by the string

$$
Q_{1}=(0, \underbrace{(1,1),(1,1), \cdots(1,1)}_{h \text { times }}) .
$$

If the structure of Stack 2 is given by $Q_{2}=(1,(1, H-3))$ then it is only possible to retrieve the top target container from Stack 1, so that the problem has no solution.

Let the structure of Stack 2 be given by

$$
Q_{2}=(1, \underbrace{(1,1),(1,1), \cdots(1,1)}_{h-1 \text { times }})
$$

so that initially there are $n=2 H-1$ containers and only one free slot, which is the top slot of Stack 2.

The only way to solve the problem is at any time to move all top non-target containers from the stack that is currently full and to retrieve the target container that becomes available. Then the same actions are performed starting from the other stack. The process is repeated until all target containers are retrieved. During this process, 1 container is moved from Stack 1 to Stack 2; then 2 containers are moved from Stack 2 to Stack 1; then 3 containers are moved from Stack 1 to Stack 2, etc. The total number of moves is given by $1+2+3+\cdots+H-1=\frac{1}{2} H(H-1)=O\left(H^{2}\right)=O\left(n^{2}\right)$. Figure 5 illustrates this process for $H=8$.

\section{The Bi-objective Block Retrieval Problem}

In this section, we discuss the computational complexity of the $2 \mathrm{BRTP}$ and a B\&B algorithm for the problem. Additionally, we also describe a beam search algorithm in order to cope with the intrinsic difficulty of the problem.

An instance of the $2 \mathrm{BRTP}$ can be described by providing the following information. There are $S \geq 2$ stacks such that stack $i$ has a height of $h_{i}$ tiers (slots) numbered from bottom to top. Define $H=\max \left\{h_{i} \mid 1 \leq i \leq S\right\}$, the maximum height. Each stack $i$ can be seen as an array such that in each cell the group $g, 1 \leq g \leq G$, of the corresponding container is stored.

A target group $t$ is given, and the primary objective is to retrieve all containers of that group with the smallest possible number of relocations. For each group $g \neq t$, a probability $P_{g}$ that the containers of that group will form the next target is given. The secondary objective function can be written as $\sum_{g \in\{1, \ldots, G\} \backslash t} P_{g} C_{g}$, where $C_{g}$ is the cost of the retrieval of group $g$ from the bay of the configuration obtained after the containers of the primary target group $t$ have been retrieved at the minimum $\operatorname{cost} C_{t}$.

\subsection{Proof of $\mathcal{N} \mathcal{P}$-Hardness for the 2BRTP}

Proposition 4.1. The 2BRTP is NP-Hard in the strong sense, even if all groups have the same probability to become the next target.

Proof Proof. The 3-Dimensional Matching (3DM) is used for reduction. Given 3 disjoint sets $X, Y$, and $Z$ with $|X|=|Y|=|Z|=n$, and a set of triplets $T \subseteq X \times Y \times Z$, find $T^{\prime} \subseteq T$ such that each element of $X, Y$, and $Z$ is contained within exactly one triplet in $T^{\prime}$. Recall that 3DM is $\mathcal{N} \mathcal{P}$-Complete in the strong sense (Garey and Johnson 1979). 


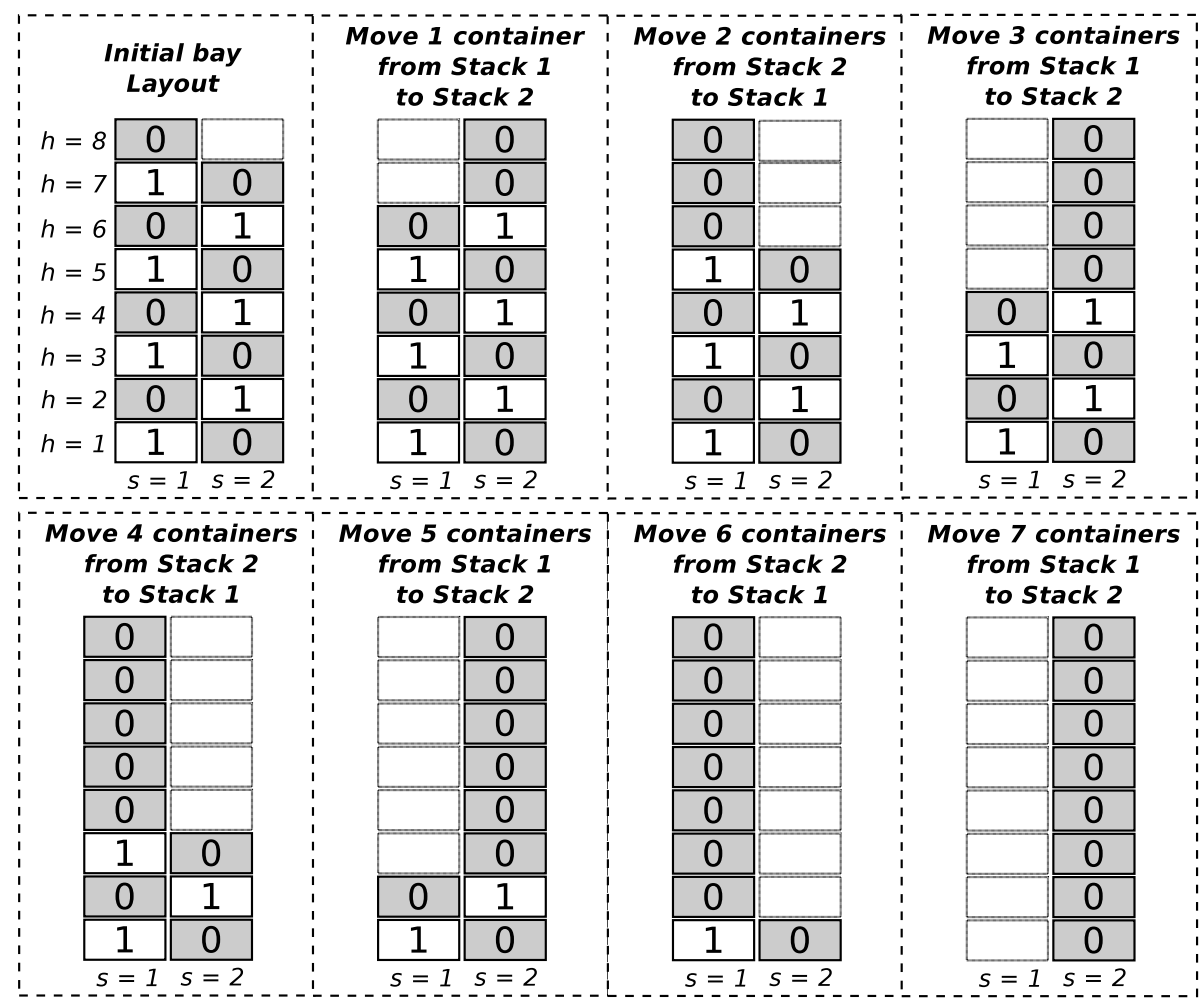

Figure 5: Solution process applied to a BRTP instance with $S=2$ and $H=8$.

Given an instance of $3 \mathrm{DM}$ with $X=\{1, \ldots, n\}, Y=\{n+1, \ldots, 2 n\}$, and $Z=\{2 n+1, \ldots, 3 n\}$, let $T$ consist of triplets $\tau_{k}, 1 \leq k \leq|T|$, and let an integer $g, 1 \leq g \leq 3 n$, occur in $\theta_{g}$ triplets of set $T$. Based on the described instance of $3 \mathrm{DM}$, we construct an instance of the $2 \mathrm{BRTP}$ with $3|T|+2$ stacks, each of height $H=3 n+3|T|+1$.

The number of groups $G$ is defined equal to $3 n+|T|+1$, and their structure is as follows. The primary target is group 0 which consists of a single container. For each group $g, 1 \leq g \leq 3 n$, there are $\theta_{g}+1$ containers. Group $3 n+1$ consists of $3|T|+1$ containers, while each group $g$, $3 n+2 \leq g \leq 3 n+|T|+1$, contains $9|T|+9 n$ containers. It is equally probable that a group $g, 1 \leq g \leq 3 n+|T|+1$, is chosen as the next target, i.e., the probability of such a choice is $P=1 /(3 n+|T|+1)$ for any $g \neq 0$.

The bay configuration is as follows. The structure of each stack is described from bottom to top. Stack 1 contains $3|T|+1$ containers of group $3 n+1$; the remaining slots of that stack are initially free. Each triplet $\tau_{k}, 1 \leq k \leq|T|$, is associated with three stacks $3 k-1,3 k$ and $3 k+1$. Each of these stacks contains containers of group $3 n+k+1$ up to height $3 n+3|T|-1$. One container of the group defined by the first, second, and third elements of triple $\tau_{k}$ is placed on top of stack $3 k-1,3 k$, and $3 k+1$, respectively. Each of the stacks from 2 to $3|T|+1$ contains one empty slot. The initial configuration of the remaining stack $3|T|+2$ is as follows: it contains one container of the target group 0 at the bottom, then blocks of three containers of the groups $3 n+2,3 n+3, \ldots, 3 n+|T|+1$ in this order, followed by one container of each group $1,2, \ldots, 3 n$ in this order, leaving no empty slots.

The generic structure of the instance is shown in Figure 6a. For illustration, Figure 6b shows the bay configuration derived for $n=3, X=\{1,2,3\}, Y=\{4,5,6\}, Z=\{7,8,9\}$, and $T=$ $\{(1,5,7),(2,4,9),(2,5,8),(3,6,8)\}$.

The length of input of $3 \mathrm{DM}$ in the unary encoding is $\mathcal{O}(n|T|)$. The total number of slots in the produced bay configuration is $\mathcal{O}\left(n|T|+|T|^{2}\right)=\mathcal{O}\left(|T|^{2}\right)$, so that the described input of 
the 2BRTP can be obtained in time that is polynomial in terms of $\mathcal{O}(n|T|)$, which provides a pseudopolynomial-time reduction.

We prove that $3 \mathrm{DM}$ has a solution if and only if for the constructed instance of the 2BRTP the value of the primary objective is at most $3|T|+3 n$, while the value of the secondary objective is $P(3 n(n+1) / 2)+6 P|T|$.

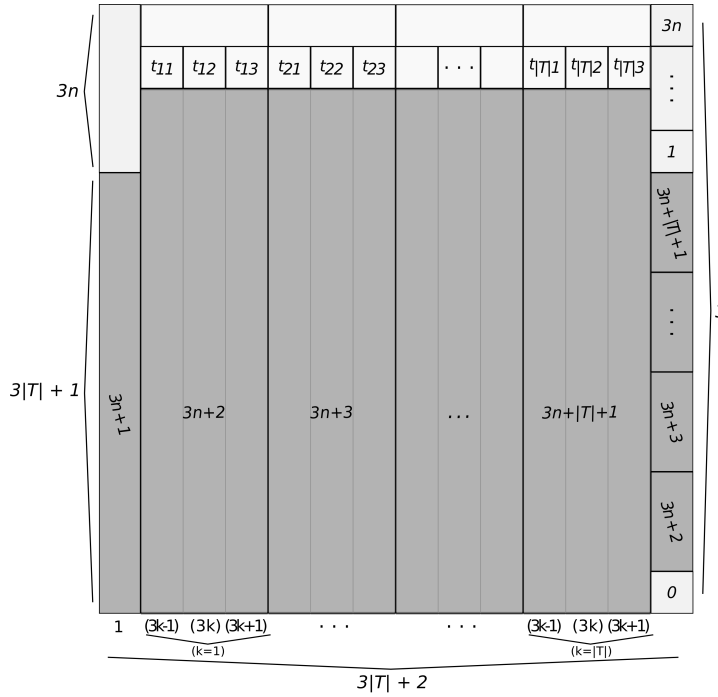

(a)

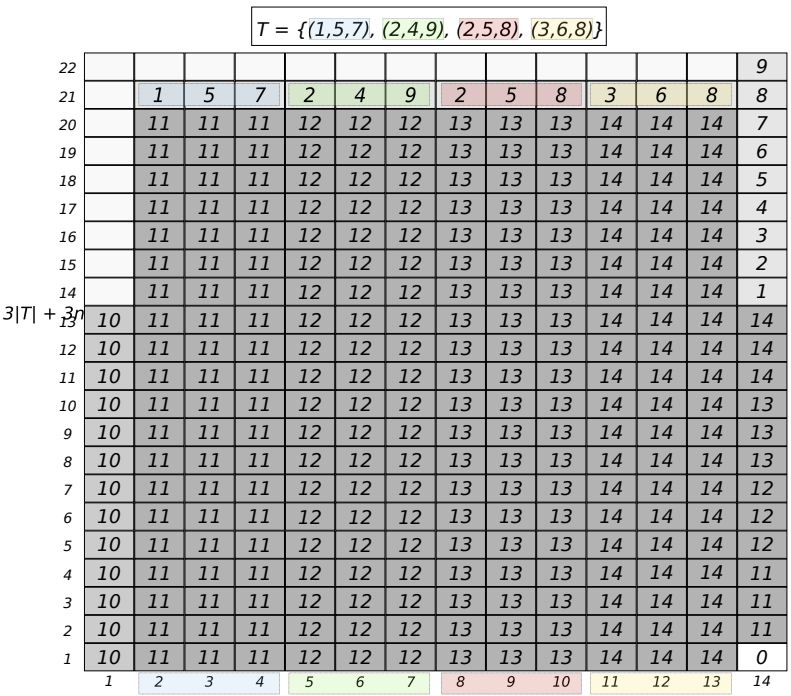

(b)

Figure 6: (a) BRTP bay arrangement for generic instances. (b) BRTP bay arrangement for the proposed example.

Necessity $(\Rightarrow)$ : Assume that $3 \mathrm{DM}$ has a solution given by a collection $T^{\prime}$ of $n$ triplets. In order to retrieve the container of the target group 0 , we need to relocate $3|T|+3 n$ other containers from stack $3|T|+2$. Thus, in our solution to the 2 BRTP we only may relocate the containers from that stack. We relocate the containers of groups 1 to $3 n$ from stack $3|T|+2$ to the top of the stacks corresponding to the triplets in $T^{\prime}$ to match the container on top. For relocating containers of groups $3 n+|T|+1$ down to $3 n+2$ from stack $3|T|+2$, we use one of the stacks $2, \ldots, 3|T|+1$ for which position $3 n+|T|+1$ is empty and the group of the container to be relocated matches that of the container at the bottom of the stack. If such a stack is not available, we relocate the container to the first stack. In the obtained configuration, stack $3|T|+2$ is empty, so that it is possible to retrieve containers of any group that may obstruct a secondary target.

As a result of the relocation process described, the first stack will be totally filled, since $3 n$ containers in total will be moved from stack $3|T|+2$. These containers belong to $n$ groups from the set $\{3 n+2, \ldots, 3 n+|T|+1\}$ and will be organized in blocks of three. Thus, a contribution of stack 1 to the secondary objective is $P(3 n(n+1) / 2)$. We add a single container on top of a stack from 2 to $3|T|+1$ so that its group either matches the group of the containers at the bottom or the group of the container on top will result in a secondary objective contribution of $2 P$, summing up to $6 P|T|$ for all such stacks. The value of the secondary function is hence $P(3 n(n+1) / 2)+6 P|T|$, as required.

Sufficiency $(\Leftarrow)$ : Assume that we have a solution to the constructed 2BRTP instance with the values of the primary and the secondary objective functions at most $3|T|+3 n$ and $P(3 n(n+1) / 2)+$ $6 P|T|$, respectively. As noticed above, the value of the primary objective must be equal to $3|T|+3 n$ and no relocations from stacks other than $3|T|+2$ are allowed.

The total number of the free slots in the initial configuration is equal to $3|T|+3 n$, i.e., in any solution feasible with respect to the primary objective, stack 1 will be filled with $3 n$ containers. The contribution of stack 1 to the secondary objective increases as the number of groups in the stack 
increases. The minimum contribution is realized if, as a result of reallocation, containers that belong to $n$ groups are moved to stack 1 and arranged there as blocks of three for each group. This results into a contribution of $P(3 n(n+1) / 2)$ from stack 1 to the secondary objective function.

For each stack from the range from 2 to $3|T|+1$, the minimum contribution towards the secondary objective is $2 P$, which is realized when the container relocated to this stack either matches the group of the container on top or the group of the containers at the bottom. Relocating a container that does not match the other containers in such a stack will result in a contribution of $3 P$. Hence, the minimum total contribution of stacks 2 to $3|T|+1$ is $6 P|T|$. The only way of attaining the total minimum of $P(3 n(n+1) / 2)+6 P|T|$ is to relocate the containers of group 1 to $3 n$ from the last stack the stacks of the range from 2 to $3|T|+1$ that correspond to exactly $n$ triplets in $T$. That means that 3DM must have a solution. Q.E.D.

Note that Proposition 4.1 does not resolve the complexity status of the special case of the 2BRTP in which each group consists of a single container. It can be verified that if each group consists of a single container and the retrieval probabilities for the secondary target are equal for all groups other than the primary target, then the 2BRTP can be solved in polynomial time. However, in the case of unequal probabilities and each group consists of a single container, the complexity status of the 2BRTP is open.

\subsection{A BEBB algorithm for the 2BRTP}

The bi-objective structure of the $2 \mathrm{BRTP}$ requires the sequential solution of the retrieval of the target container group, as well as all scenarios regarding the retrieval of the remaining groups. Hence, developing an Integer Programming model for 2BRTP is unlikely to yield a tractable formulation. In what follows, we derive a combinatorial bound and a B\&B algorithm to solve the 2BRTP.

A lower bound for the secondary 2BRTP objective function approximates the expected number of relocations to retrieve the non-target groups. The bound is based on the concept of counting the obstructing containers above any non-target group and below all target containers in the stack. Figures 7 (a) and (b) depict the container deadlocks that will be taken into account when computing the lower bounds for the primary objective and for the secondary objective, respectively. In Figure 7 (b), the stack $s=1$ does not contain any target container and thus none of its containers are going to be relocated during the retrieval process of the target group. The same observations can be made about those non-target containers positioned below target containers, as in stack $s=2$. Nevertheless, once the retrieval process is finished, the containers belonging to group 2 or group 3 will in turn be considered for retrieval and in both cases additional relocations will be necessary. If group 2 is the second target, at least one container of group 3 will be relocated. Similarly, if group 3 is the second target, at least 4 containers of group 2 will be relocated. Denoting the probability of retrieving group $g$ as $P_{g}$, the secondary objective lower bound for the bay depicted will be $E[R e l]=\left(1 \times P_{2}\right)+\left(4 \times P_{3}\right)$. Containers above any of the target containers cannot be taken into account in the secondary objective computation, given that their relocation may alter the bay configuration.

The pseudocode in Algorithm 3 states the steps required for computing the lower bound for the secondary objective of 2 BRTP. For each container group in the bay other than the target, the algorithm counts the number of containers of non target groups located above it in all stacks, if no target container is located in between. These containers will be deadlocks in the secondary objective computation and are therefore added to the lower bound. Note that for each non target group and stack, we compute the bound based on the topmost container and stacks containing one container or no containers do not contribute to the lower bound.

We now present our B\&B algorithm for the $2 \mathrm{BRTP}$, the pseudocode of which is provided in Algorithm 4. Before executing the $\mathrm{B} \& \mathrm{~B}$, a preprocessing step is performed in the initial bay in order to retrieve all the unobstructed target containers (i.e., in the top tiers). In addition, the optimal number or relocations for the BRTP and the lower and upper bound for the secondary objective are also computed using Algorithms 2 and 3. The upper bound is computed by applying Algorithm 2 on the initial configuration, and then $G-1$ times on the resulting configuration, each time assuming a different $g \in\{1, \ldots, G\} \backslash\{t\}$ to be the group to be retrieved second. The 


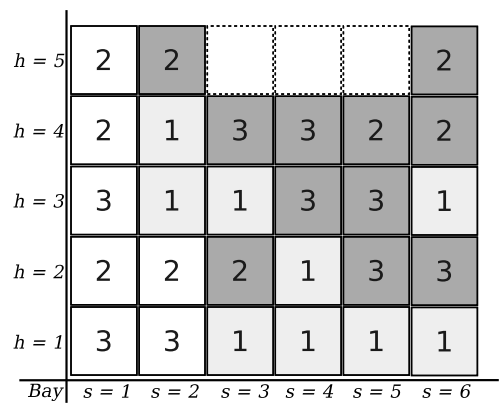

(a)

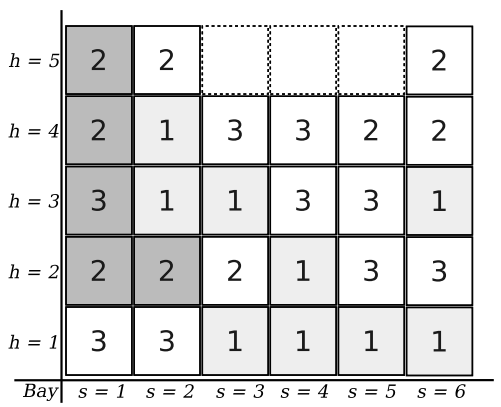

(b)

Figure 7: (a) Target group 1 and respective obstructing containers (in dark gray) which represents the primary objective lower bound, (b) Non-target groups 2 and 3 are used in the secondary objective lower bound.

overall upper bound is computed by multiplying the number of deadlocks of each group by the probability of retrieval and summing up the contribution of all groups (other than the target). The root node is initialized with the initial configuration of the bay, and the branching strategy consists of enumerating all possible retrieval operations starting from the given bay configuration (and corresponding relocations). Algorithm 4 explores the B\&B search tree in a depth first fashion. At each node of the tree, if the number of relocations executed so far for retrieving the target block is greater than the optimal BRTP solution or the lower bound is greater than or equal to the upper bound on the secondary objective, the node is fathomed.

The branching strategies employed in the BRTP B\&B algorithm described in Section 3.3 cannot be applied when solving the 2BRTP, provided they may cut off the optimal solutions. Hence, for the 2BRTP, if any target container sits in the bay and no fathoming condition is reached, new nodes are created from a B\&B node by selecting each stack $s \in\{1, \ldots, S\}$ that contains at least one target container. If the target containers in $s$ are not obstructed, they are retrieved. Otherwise, if there are containers obstructing the target containers in the selected stack, any other stack $s^{\prime} \in\{1, \ldots, S\}, s^{\prime} \neq s$, is selected and the topmost container from $s$ is relocated to $s^{\prime}$.

If the number of relocations is equal to the BRTP optimal solution, the lower bound on the secondary objective is smaller than the current upper bound, and there is no target container left in the bay, then a new best known 2BRTP solution was found. The incumbent solution is updated and the node is fathomed.

\subsection{A Beam Search algorithm for the 2BRTP}

The B\&B algorithm described above can be very time consuming depending on the size of the instance being solved. In search for a better balance between solution quality and execution time, we incorporated in Algorithm 4 the two restricted branching rules employed in the BRTP B\&B algorithm. These rules transform the B\&B algorithm into a beam search algorithm since the optimal solution can be disregarded in the search, but the CPU time requirement decreases significantly. We refer the interested reader to Furcy and Koenig (2005), Zhou and Hansen (2005) for more information about beam search algorithms. We state our branching strategy as the following two rules:

- Rule 01 (Cyclic relocations). If a stack $s$ containing target containers was selected in an iteration and the target containers in $s$ are still obstructed, choose $s$ to relocate another deadlock in the next iteration.

- Rule 02 (Non-target stacks). Select a stack $s^{\prime} \in R$ (set of stacks with no target containers), if $R \neq \emptyset$, to receive a relocated non-target container. 


\section{Computational Experiments}

The proposed algorithms were coded in $\mathrm{C}$ and executed on an Intel@ Core ${ }^{\mathrm{TM}}$ i7-2600 $3.40 \mathrm{GHz}$ CPU, with 8.0 GB of RAM memory running under GNU/Linux Debian 7.9.

\subsection{Test Instances}

In order to assess the performance of the proposed algorithms, we have transformed the set of BRP instances proposed by Caserta et al. (2012) into BRTP instances. The original BRP set is composed of 840 instances forming 21 classes, each one containing 40 instances. The classes are characterized by the dimensions of the bay $(S \times H)$. For each bay, the first $H^{\prime}=H-2$ tiers are filled with containers, resulting in a total of $n=S \times H^{\prime}$ containers. Each one of these $n$ containers is randomly placed in the bay and assigned an identifier in the range 1 to $n$, meaning that each bay is composed by $n$ groups and each group contains a container. The number of stacks is chosen among $S \in\{3,4, \ldots, 10\}$ and the number of filled tiers among $H^{\prime} \in\{3,4,5,6,10\}$. Note that not all combinations of these values were considered in Caserta et al. (2012), in which the authors point out that these bay settings are based on the physical limitations of gantry cranes. The BRP instances were transformed into BRTP instances using the original bay dimensions and amount of occupied tiers per stack. In order to fill the originally occupied space with containers, the following steps are repeated for each stack $s$ until $H^{\prime}$ tiers have been used. A group $g$, and a number of containers $h^{\prime}$ smaller than or equal to $H^{\prime}$ are selected at random and inserted in $s$. If $h^{\prime}<H^{\prime}$ the previous step is repeated until the remaining $\left(H^{\prime}-h^{\prime}\right)$ tiers have been filled. Else, the next available stack is processed. Once all stacks are processed in this manner, if there is any group not being used, the whole algorithm is repeated. This procedure was used to generate 2520 instances with $G \in\{3,4,5\}$. Note that by construction all instances in this set satisfy the condition to apply Algorithm 2.

We have also developed a random BRTP instance generator which, along with a set of chosen parameters, is used for generating additional instances. The pseudocode for the generator is shown in Algorithm 5. The procedure generates a bay with dimensions $(S \times H)$ randomly filled with containers belonging to $G$ groups. The total amount of containers is proportional to the size of the bay and an occupancy rate parameter. A random group $g \in\{1, \ldots, G\}$ and a random amount $h^{\prime}$ of containers (smaller than or equal to $H$ ) belonging to this group are selected and inserted in a stack. These steps are repeated for each stack until they are all completely filled. Next, containers are randomly removed from the bay until the occupancy rate is reached. If the container being removed is in between filled tiers, the topmost containers are moved downwards. Finally, the algorithm halts if at least one container of each group is present in the final bay, otherwise the instance is rejected and the whole process is repeated.

The procedure described above was used to generate 3240 instances with $S \in\{4,6,8,10\}, H \in$ $\{4,5,6\}$, and $G \in\{3,4,5\}$. For each combination of the these three parameters, 30 instances were generated for the occupancy rates of $70 \%, 75 \%$, and $80 \%$, resulting in 3240 instances. During the experiments, group number 1 was designated to be the target. A CPU time limit of 5 minutes was imposed for each instance. All these instances satisfied the condition to apply Algorithm 2 (we also increased the occupancy rate to $85 \%, 90 \%$ and $95 \%$ but the result was the same).

We therefore generated instances with the following artificial characteristics: $S=10, H \in$ $\{10,15,20\}, G=3$ and occupancy rate $95 \%$. These bay configurations do not respect standard height of stacks in the landside of a container terminal, but this, combined with a very high occupancy rate, decreases the likelihood of finding stacks in which the bottommost target container can be retrieved. Moreover, we slightly modified the instance generator procedure to further increase the chances of producing infeasible instances for the linear algorithm, by imposing in Line 20 that new_Containers $=1$. This would result in instances in which many pairs are in the same stack. Among 3000 generated instances, 19/22/62 did not respect condition (2) for $H \in\{10,15,20\}$, respectively. Among these, all $H \in\{10,15\}$ were solved in less than one second by the B\&B (few were the feasible retrievals in the bay), but one instance that required 57 seconds. The latter's optimal solution was 122 relocations, therefore a configuration that would require a couple of hours for the retrieval of the containers (assuming one relocation might take 1 minute) and therefore not realistic 
in real world settings. Similarly, the B\&B failed to converge within the time limit in the instances with $H=20$ due to the very large number of relocations.

\subsection{Results of instances with equal retrieval probabilities}

The following column headings are employed in Tables 1-11. Average results are reported for each instance class and set.

- Instance Set: specifies the instance set.

- OccRate: the occupancy rate employed in the respective instance set.

- Rel: the average number of relocations.

- $E[R e l]:$ the average expected number of relocations for retrieving the non-target groups

- Time(s): the average computational time in seconds.

- Gap (\%): the gap between the average expected number of relocations of non-target containers considering the final configuration obtained at the end of Algorithm 2 and the average expected number of relocations obtained using the B\&B algorithm, computed as $G a p(\%)=(100 \times$ (BRTP E[Rel] - B\&B E[Rel])/B\&B E[Rel]).

- BGap(\%): the gap between the E[Rel] obtained by the B\&B algorithm (B\&B E[Rel]), if the instance is solved to optimality, otherwise, the 2BRTP secondary objective lower bound (2BRTP LB) is employed, and the $E[R e l]$ obtained using one of the beam search algorithms (Beam E[Rel]), computed as $B G a p(\%)=(100 \times($ Beam E[Rel] $-\mathrm{B} \& \mathrm{~B} E[$ Rel $]) / \mathrm{B} \& \mathrm{~B} E[\mathrm{Rel}])$ in the case of $\mathrm{B} \& \mathrm{~B}$ optimality, and as $B G a p(\%)=(100 \times($ Beam E[Rel $]-2 \mathrm{BRTP}$ LB $) / 2 \mathrm{BRTP}$ LB $)$, otherwise.

- Solved: the number of instances that the relevant algorithm was able to solve to optimality within the time limit.

- Less 1s: the number of instances, among the solved ones, that the respective algorithm was able to solve in less than one second.

- Optimal: the number of solved instances in which the beam search algorithm was able to find the optimal solution obtained using the B\&B algorithm.

Before presenting a more detailed analysis of the computational results for the $2 \mathrm{BRTP} \mathrm{B} \& \mathrm{~B}$ and beam search algorithms, we will analyze the performance of the BRTP linear algorithm and the BRTP B\&B algorithm. Table 1 presents the results for the BRTP linear time algorithm and the BRTP B\&B algorithm. In terms of computational time, both the BRTP linear time algorithm and the $\mathrm{B} \& \mathrm{~B}$ are able to solve all the BRTP instances to optimality in less than one second.

The linear time algorithm and the $\mathrm{B} \& \mathrm{~B}$ algorithm can be used to find upper bounds for the 2BRTP, if used sequentially. The algorithms first are employed to generate a bay configuration in which all target containers are retrieved (with minimum cost), and then the same algorithms can be used to compute the cost of retrieval of all other groups.

Table 1 compares the performance of these upper bounds (the one using sequentially the linear time algorithm and the BRTP B\&B, respectively). On average, the upper bounds provided by the BRTP linear time algorithm are at least $20 \%$ better than those of the BRTP B\&B. The better upper bounds have some impact when solving the 2BRTP. The 2BRTP B\&B using the BRTP linear time algorithm was able to solve 3 instances more than the 2BRTP B\&B using the BRTP B\&B within the time limit, and on average they have similar computational times. Provided the good quality of the results obtained by the BRTP linear time algorithm, in the remaining of the computational experiments, we will be using the linear time algorithm algorithm as heuristic 2BRTP solver.

Table 1 shows that the $\mathrm{B} \& \mathrm{~B}$ algorithm is capable of solving 1077, 1067, and 1074 out of 1080 instances within the specified time limit, respectively. In addition, $98 \%$ of the instances were solved 
in less than one second. We note that the $\mathrm{B} \& \mathrm{~B}$ algorithm results in solutions with a higher $\mathrm{E}[\mathrm{Rel}]$ value than the linear time algorithm, due to the depth first implementation that tends to stack the containers belonging to other customers into the leftmost columns. Tables 2, 3, 4 present detailed results for the 2BRTP B\&B algorithm using the BRTP linear time algorithm in bays of different dimensions, variable number of groups, and occupancy rate of $70 \%, 75 \%$, and $80 \%$, respectively.

The results of both BRTP and 2BRTP for the instances of Caserta et al. (2012) with different number of groups are presented in Table 5. The B\&B method is capable of solving $94 \%$ of the instances within the specified time limit, and $92 \%$ of these instances were solved in less than one second. The $\mathrm{B} \& \mathrm{~B}$ algorithm requires a longer $\mathrm{CPU}$ time to prove optimality when the number of relocations increases. Nevertheless, most of the instances with up to 9 stacks and 7 tiers were solved to optimality. The BRTP linear time algorithm requires less than one second of CPU time for each instance.

In summary, the B\&B algorithm solved $97 \%$ of the instances to optimality. We observe that 2BRTP allows for average savings in the number of expected number of operations at least of $19 \%$ across all instances considered, with respect to solutions obtained using BRTP algorithms sequentially. This highlights the importance of looking ahead and trying to achieve the best possible bay configuration in the process of retrieving the target containers.

\subsubsection{Results of Beam Search Algorithms}

Table 6 presents the average results for three versions of the beam search method described in Section 4.3. The first version applies the first branching rule, the second applies the second rule, and the third employs both rules. The results obtained are compared with those reported in the previous tables for the B\&B algorithm. Summing up for the two instance sets, the beam search employing Rule 01 is capable of solving $99 \%$ of the instances within the time limit, $98 \%$ of those solved in less than 1 second, and for only 5 instances the method was not able to find the known optimal solutions. The beam search employing Rule 02 was not capable of solving only seven instances within the time limit, 99\% were solved in less than 1 second, and all the known optimal solutions were found. The beam search employing both rules was capable of solving all but 2 instances within the time limit, and only 4 instances were not solved in less than 1 second. The method successfully found the known optimal solutions for all instances but 5, similar to the method using Rule 01. The reason is that the third method inherits the limitations of both rules. Among the three, the method combining the two rules performs better both in terms of computing time and solution quality. We attribute the success of the beam search algorithm to the choice of intuitive branching rules.

\subsection{Results for instances with unequal retrieval probabilities}

The results obtained with the BRTP linear time algorithm and the 2BRTP B\&B algorithms for the generated instances with different retrieval probabilities for the groups, and occupancy rates of $70 \%, 75 \%$, and $80 \%$ are similar to those obtained when using equal retrieval probabilities. They are presented in Tables 7, 8, 9 of Appendix B. The B\&B algorithm is capable of solving 1076, 1065, and 1073 out of 1080 instances within the specified time limit, respectively. In addition, $98 \%$ of the instances were solved in less than one second.

The results of both BRTP and 2BRTP for the instances of Caserta et al. (2012) with different number of groups and different retrieval probabilities per group are presented in Table 10 of Appendix B. The B\&B method is capable of solving $94 \%$ of the instances within 5 CPU minutes, and $91 \%$ of those were solved in less than one second. Similar to the case with equal retrieval probabilities, the average number of expected relocations, when solving them as a 2BRTP, was reduced by $21 \%$ across all instances considered, with respect to the BRTP optimal solutions.

The average results for the three versions of the beam search method are summarized in Table 11 of the appendix. Among the three methods, the beam search combining the two rules outperforms the other two both in terms of time and solution quality. 


\section{Concluding Remarks}

In this paper, we study two problems related to the optimal container retrieval, the BRTP and the 2BRTP. We show that both problems are in general NP-hard, however, the BRTP can be solved in polynomial time, provided that the number of stacks is fixed. For the BRTP, a B\&B algorithm is designed, as well as and a linear time algorithm that is able to handle instances of practical relevance. For the 2BRTP, a B\&B algorithm and a beam search algorithm are developed.

The effectiveness of the 2BRTP B\&B and beam search algorithms has been assessed by extensive computational experiments on two benchmark sets with instance bay sizes varying from small to large dimensions. The B\&B algorithm is capable of solving $97 \%$ of the instances to optimality within 5 CPU minutes, and the average number of expected relocations has been reduced by at least $21 \%$ with respect to the BRTP optimal solutions. Three versions of the beam search algorithm that employ different branch rules have been tested. The most efficient version is capable of solving $99.9 \%$ of the instances within the time limit. The reduction in the average number of expected relocations varies among $1 \%$ and $2 \%$ when compared to the results obtained by the B\&B algorithm. The 2BRTP instances with up to 10 stacks in a bay with a maximum height of 6 and 5 groups of customers can be solved consistently to optimality within 5 minutes of CPU time.

Further research may focus on multiple bays, and optimizing the expected number of relocations when more information about the sequence of retrieval for the non-target containers is available.

\section{Appendices}

\section{A. Pseudocode Notation and Data Structures}

In the pseudocodes shown in Algorithms 1, 2, 3, 4, and 5, the following data structures, functions, and variables are employed.

- accounted_Group is an array of size $G$. It contains the information regarding if a given group appears or not in a stack.

- aux is an auxiliary variable used to identify a specific slot of a stack or array.

- available_Tiers is a variable that contains the number of free tiers/slots in a stack.

- bay_configuration is a matrix of dimensions $(S \times H)$. It holds the current configuration of the container bay.

- bound is a variable that contains the value of the bound of the incumbent 2BRTP solution.

- blocks_left is a variable that stores how many target containers are still in the bay and need to be retrieved.

- BRTP_relocations is a variable that contains the number of relocations need retrieve all the target containers from a given bay (i.e., the BRTP solution).

- depth is a variable that stores the level of the branch-and-bound tree being explored.

- $\operatorname{find}()$ is a function that informs if a given stack contains a given target container.

- first_Element () is a function that informs the first valid element from an array.

- free_stack is a variable used to identify a stack that does not contain target blocks and has empty slots.

- group_id is a variable used to identify the group for which containers will be generated. 
- $\operatorname{insert}()$ is a function used to insert new elements in the arrays sorted_StackList and priority_StackList. The new elements are inserted after the last used position.

- lower_bound is a variable that stores the value of the best lower bound.

- new_Containers is a variable that informs the amount of containers that will be inserted for the selected group.

- number_Container is a variable that informs the number of containers to be inserted in the bay being generated.

- occ_Rate is a variable that stores the occupancy rate of a bay.

- pickup_stack is a variable that keep track of the stack from where non target containers have to be relocated in order to access the target containers being obstructed.

- $\operatorname{pr}()$ is a function that informs the probability of a given container group.

- priority_StackList is an array of size $S$. It stores the information about which stacks does not contain target containers and has available slots.

- relocations is a variable used to count the number of relocations performed during the retrieval process performed by the algorithms.

- remove() is a function used to remove an element from the array specified.

- retrieve() is a function used to retrieved a target container from the bay slot specified by the parameters. It updates the affected data structures; e.g., bay_configuration.

- second_bound is an auxiliary variable employed during the computation of the secondary objective in a 2BRTP solution.

- seed is a variable used to initialize the random number generator.

- size() is a function that informs the size of an array; i.e., it informs the number of elements that an array contains.

- slot is a variable used to identify a specific slot of a stack or array.

- sorted_StackList is an array of size S. It stores the information about the stacks that contains target containers and it is sorted according to the number of deadlock in each stack.

- Target_block_LB() is a function that computes the number of deadlocks for a given bay and target group.

- target_container is a variable that keep track of the current target group.

- upper_bound is a variable that stores the value of the best upper bound.

- used_Tiers is a variable that contains the number of occupied tiers/slots in a stack. 


\section{B. Results for instances with unequal retrieval probabilities}

During our experiments with unequal retrieval probabilities, we generate the probabilities $P_{g}$, for each group $g \in\{1, \ldots, G\}, g \neq t$ as follows. For the first group, the probability is set to be a random number in $\left.r_{1}=\right] 0.0,1$ [, for the second group the probability is a random number in $] 0.0,1-r_{1}[$, and we similarly assign the probabilities to the other groups, but the last. For the latter, the probability is set to $1-\sum_{i=1, \ldots, G-1} r_{i}$.

Tables 7, 8, 9 present the B\&B results of both BRTP and 2BRTP for the generated instances with different retrieval probabilities for the groups, and occupancy rates of $70 \%, 75 \%$, and $80 \%$.

The results of both BRTP and 2BRTP for the instances of Caserta et al. (2012) with different number of groups and different retrieval probabilities per group are presented in Table 10. The average results for the three versions of the beam search method are summarized in Table 11.

\section{Results for BRP algorithm}

\section{References}

S. Borjian, V. Galle, V. H. Manshadi, C. Barnhart, and P. Jaillet. Container relocation problem: Approximation, asymptotic, and incomplete information. CoRR, abs/1505.04229, 2015a. URL http://arxiv.org/abs/1505.04229.

S. Borjian, V. H. Manshadi, C. Barnhart, and P. Jaillet. Managing relocation and delay in container terminals with flexible service policies. CoRR, abs/1503.01535, 2015b. URL http://arxiv.org/abs/ 1503.01535.

H. J. Carlo, I. F. A. Vis, and K. J. Roodbergen. Seaside operations in container terminals: literature overview, trends, and research directions. Flexible Services and Manufacturing Journal, 27(2):224-262, 2013.

H. J. Carlo, I. F. A. Vis, and K. J. Roodbergen. Storage yard operations in container terminals: Literature overview, trends, and research directions. European Journal of Operational Research, 235(2):412-430, 2014a.

H. J. Carlo, I. F. A. Vis, and K. J. Roodbergen. Transport operations in container terminals: Literature overview, trends, research directions and classification scheme. European Journal of Operational Research, 236(1):1-13, 2014b.

M. Caserta and S. Voß. A corridor method-based algorithm for the pre-marshalling problem. In M. Giacobini, A. Brabazon, S. Cagnoni, G. A. Di Caro, A. Ekárt, A. I. Esparcia-Alcázar, M. Farooq, A. Fink, and P. Machado, editors, Applications of Evolutionary Computing, volume 5484 of Lecture Notes in Computer Science, pages 788-797. Springer Berlin Heidelberg, 2009.

M. Caserta, S. Schwarze, and S. Voß. Container rehandling at maritime container terminals. In J. W. Böse, editor, Handbook of Terminal Planning, volume 49 of Operations Research/Computer Science Interfaces Series, pages 247-269. Springer New York, 2011.

M. Caserta, S. Schwarze, and S. Voß. A mathematical formulation and complexity considerations for the blocks relocation problem. European Journal of Operational Research, 219(1):96-104, 2012.

B. Castilho and C. F. Daganzo. Handling strategies for import containers at marine terminals. Transportation Research Part B: Methodological, 27(2):151-166, 1993.

T. H. Cormen, C. E. Leiserson, R. L. Rivest, and C. Stein. Introduction to Algorithms, Third Edition. The MIT Press, Cambridge, Massachusetts, USA, 3rd edition, 2009.

C. F. Daganzo. The crane scheduling problem. Transportation Research Part B: Methodological, 23(3): 159-175, 1989.

R. Dekker, P. Voogd, and E. van Asperen. Advanced methods for container stacking. OR Spectrum, 28(4): 563-586, 2006.

P. Flajolet and R. Sedgewick. Analytic Combinatorics. Cambridge University Press, New York, NY, USA, 1 edition, 2009

D. Furcy and S. Koenig. Limited discrepancy beam search. In Proceedings of the 19th International Joint Conference on Artificial Intelligence, IJCAI'05, pages 125-131, 2005. 
M. R. Garey and D. S. Johnson. Computers and Intractability: A Guide to the Theory of NP-Completeness. W. H. Freeman \& Co., New York, NY, USA, 1979.

A. H. Gharehgozli, G. Laporte, Y. Yu, and R. de Koster. Scheduling twin yard cranes in a container block. Transportation Science, 49(3):686-705, 2015.

K. H. Kim. Evaluation of the number of rehandles in container yards. Computers 8 Industrial Engineering, 32(4):701-711, 1997.

K. H. Kim and G.-P. Hong. A heuristic rule for relocating blocks. Computers 85 Operations Research, 33 (4):940-954, 2006.

K. H. Kim and H. B. Kim. Segregating space allocation models for container inventories in port container terminals. International Journal of Production Economics, 59(1-3):415-423, 1999.

K. H. Kim and K. T. Park. A note on a dynamic space-allocation method for outbound containers. European Journal of Operational Research, 148(1):92-101, 2003.

D. $\mathrm{Ku}$ and T. S. Arthanari. Container relocation problem with time windows for container departure. European Journal of Operational Research, 252(3):1031-1039, 2016.

C.-Y. Lee and D.-P. Song. Ocean container transport in global supply chains: Overview and research opportunities. Transportation Research Part B: Methodological, 95:442-474, 2017.

J. Lehnfeld and S. Knust. Loading, unloading and premarshalling of stacks in storage areas: Survey and classification. European Journal of Operational Research, 239(2):297-312, 2014.

M. Liu, C.-Y. Lee, Z. Zhang, and C. Chu. Bi-objective optimization for the container terminal integrated planning. Transportation Research Part B: Methodological, 93, Part B:720-749, 2016.

R. Roberti and D. Pacino. A decomposition method for finding optimal container stowage plans. Operations Research, 2016. submitted for publication.

D. Steenken, S. Voß, and R. Stahlbock. Container terminal operation and operations research - a classification and literature review. OR Spectrum, 26(1):3-49, 2004.

S. Tanaka and K. Takii. A Faster Branch-and-Bound Algorithm for the Block Relocation Problem. IEEE Transactions on Automation Science and Engineering, 13(1):181-190, 2016.

I. F. A. Vis and K. J. Roodbergen. Scheduling of container storage and retrieval. Operations Research, 57 (2):456-467, 2009.

I. Watanabe. Characteristics and analysis method of efficiencies of container terminal - an approach to the optimal loading/unloading method. Container Age, pages 36-47, 1991.

E. Zehendner, M. Caserta, D. Feillet, S. Schwarze, and S. Voß. An improved mathematical formulation for the blocks relocation problem. European Journal of Operational Research, 245(2):415-422, 2015.

R. Zhou and E. A. Hansen. Beam-stack search: Integrating backtracking with beam search. In In International Conference on Automated Planning and Scheduling (ICAPS), pages 90-98, 2005. 


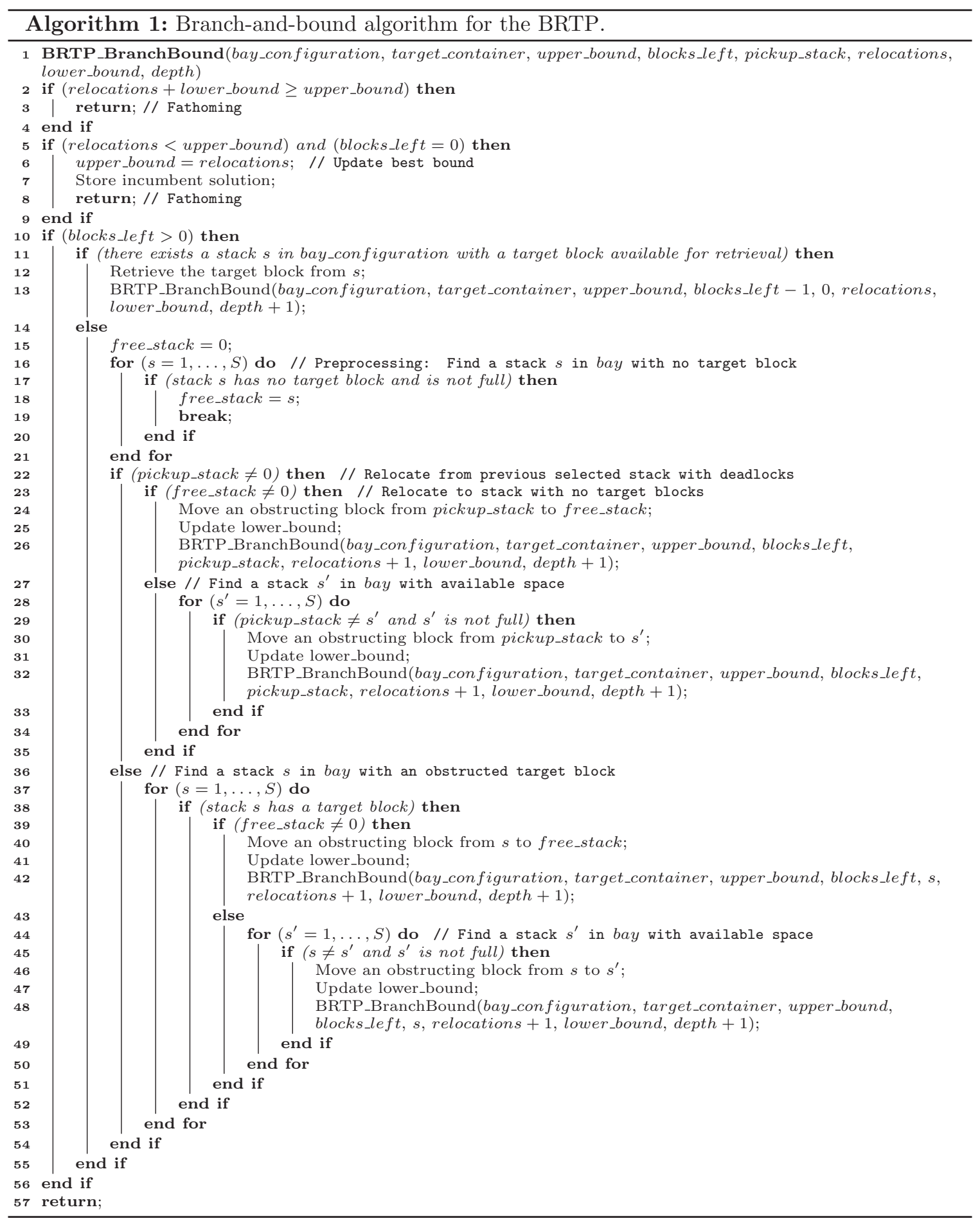



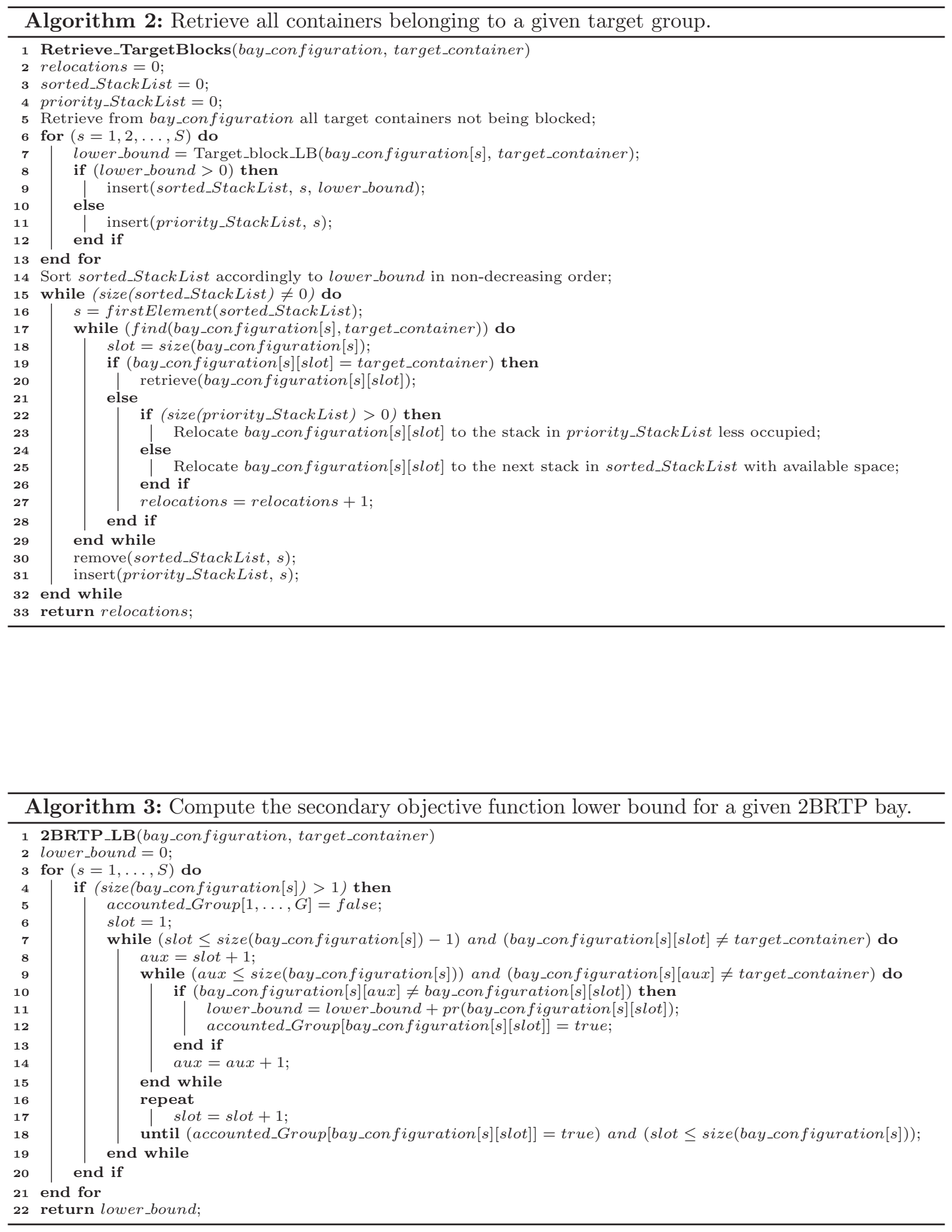


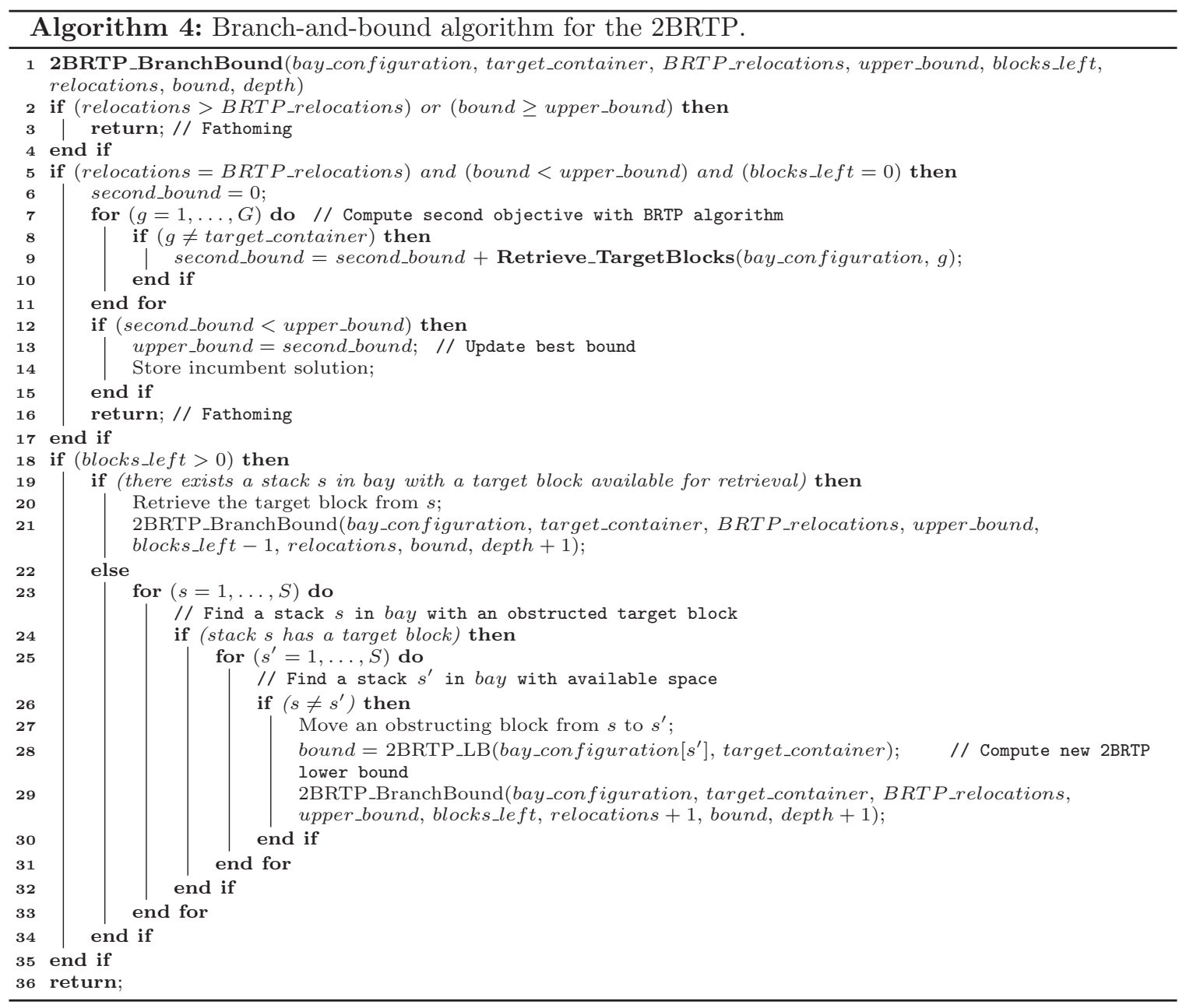




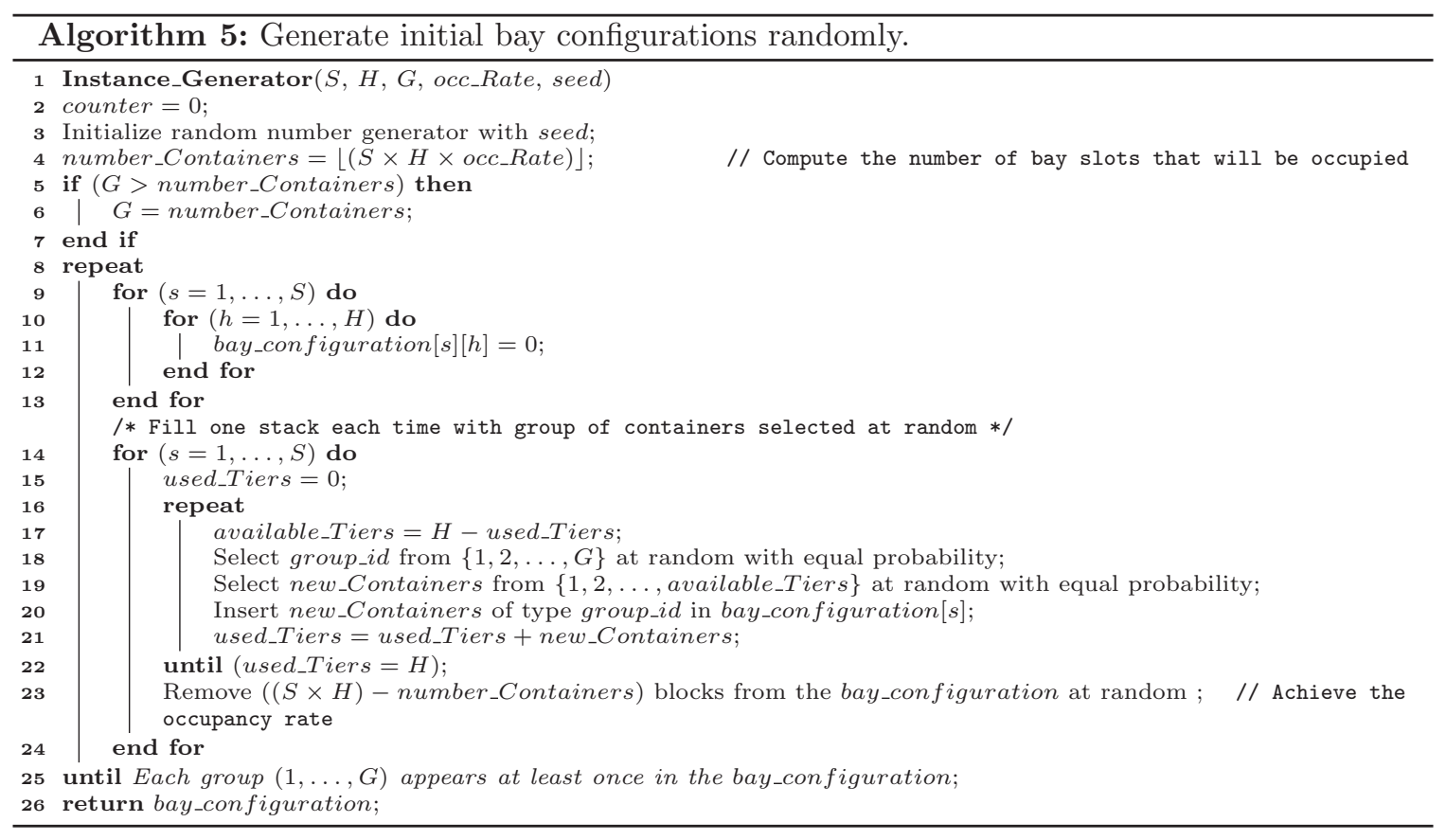

Table 1: Results of the BRTP linear time and B\&B algorithms on equal retrieval probabilities instances.

\begin{tabular}{|c|c|c|c|c|c|c|c|c|c|c|c|}
\hline & & \multicolumn{4}{|c|}{ BRTP linear } & \multicolumn{6}{|c|}{ BRTP B\&B } \\
\hline Instance Set & OccRate & Rel & $\mathrm{E}[\mathrm{Rel}]$ & Solved & Time(s) & Rel & $\mathrm{E}[\mathrm{Rel}]$ & Rel Gap(\%) & Gap(\%) & Solved & Time(s) \\
\hline \multirow{3}{*}{ BRTP } & $70 \%$ & 2.08 & 1.38 & 1077 & 0.37 & 2.08 & 1.66 & 0.00 & -22.18 & 1078 & 0.54 \\
\hline & $75 \%$ & 2.40 & 1.55 & 1067 & 0.27 & 2.40 & 1.84 & 0.00 & -22.16 & 1066 & 0.40 \\
\hline & $80 \%$ & 2.43 & 1.75 & 1074 & 0.71 & 2.43 & 2.00 & 0.00 & -20.32 & 1073 & 0.50 \\
\hline Caserta et al. (2012) & $60 \%-85 \%$ & 3.22 & 2.32 & 2387 & 1.32 & 3.22 & 2.69 & 0.00 & -21.20 & 2385 & 1.28 \\
\hline & & & & \multicolumn{2}{|c|}{ 2BRTP B\&B } & & & & & \multicolumn{2}{|c|}{ 2BRTP B\&B } \\
\hline
\end{tabular}


Table 2: Results of the BRTP linear time and 2BRTP B\&B algorithms for 2BRTP instances, occupancy rate $70 \%$.

\begin{tabular}{rrr|rr|rrrr}
\hline & & \multicolumn{5}{|c|}{ BRTP linear } & \multicolumn{3}{c}{ BRTP B\&B } \\
$S$ & $H$ & $G$ & Rel & E[Rel] & E[Rel $]$ & Time(s) & Gap $(\%)$ & Solved \\
\hline 4 & 4 & 3 & 0.47 & 0.60 & 0.57 & 0.00 & 3.45 & 30 \\
4 & 4 & 4 & 0.93 & 0.67 & 0.57 & 0.00 & 17.53 & 30 \\
4 & 4 & 5 & 0.87 & 0.83 & 0.79 & 0.00 & 9.44 & 30 \\
4 & 5 & 3 & 1.40 & 0.77 & 0.60 & 0.00 & 15.12 & 30 \\
4 & 5 & 4 & 1.30 & 1.19 & 0.99 & 0.00 & 36.21 & 30 \\
4 & 5 & 5 & 1.30 & 1.08 & 0.98 & 0.00 & 12.42 & 30 \\
4 & 6 & 3 & 2.03 & 1.28 & 1.07 & 0.00 & 17.90 & 30 \\
4 & 6 & 4 & 1.43 & 1.16 & 1.11 & 0.00 & 1.72 & 30 \\
4 & 6 & 5 & 1.37 & 1.33 & 1.13 & 0.00 & 29.34 & 30 \\
\hline 6 & 4 & 3 & 0.93 & 0.52 & 0.48 & 0.00 & 1.72 & 30 \\
6 & 4 & 4 & 1.23 & 0.94 & 0.82 & 0.00 & 18.51 & 30 \\
6 & 4 & 5 & 0.97 & 0.70 & 0.63 & 0.00 & 12.64 & 30 \\
6 & 5 & 3 & 2.30 & 0.90 & 0.70 & 0.00 & 16.03 & 30 \\
6 & 5 & 4 & 3.13 & 1.24 & 0.96 & 0.06 & 32.65 & 30 \\
6 & 5 & 5 & 1.17 & 1.33 & 1.21 & 0.00 & 18.94 & 30 \\
6 & 6 & 3 & 3.40 & 1.48 & 0.87 & 0.05 & 55.00 & 30 \\
6 & 6 & 4 & 2.90 & 1.69 & 1.43 & 0.01 & 29.94 & 30 \\
6 & 6 & 5 & 1.67 & 1.78 & 1.62 & 0.00 & 15.80 & 30 \\
\hline \hline 8 & 4 & 3 & 1.83 & 0.75 & 0.68 & 0.00 & 4.31 & 30 \\
8 & 4 & 4 & 1.83 & 0.98 & 0.87 & 0.01 & 14.83 & 30 \\
8 & 4 & 5 & 1.20 & 1.05 & 0.98 & 0.00 & 14.58 & 30 \\
8 & 5 & 3 & 2.23 & 1.53 & 1.35 & 0.00 & 8.95 & 30 \\
8 & 5 & 4 & 2.27 & 1.72 & 1.52 & 0.06 & 20.99 & 30 \\
8 & 5 & 5 & 2.13 & 1.68 & 1.45 & 0.01 & 25.83 & 30 \\
8 & 6 & 3 & 3.37 & 1.97 & 1.53 & 4.95 & 27.96 & 30 \\
8 & 6 & 4 & 3.17 & 1.68 & 1.22 & 0.08 & 57.33 & 30 \\
8 & 6 & 5 & 2.40 & 2.09 & 1.88 & 0.01 & 24.96 & 30 \\
\hline \hline 10 & 4 & 3 & 2.20 & 1.30 & 1.18 & 0.00 & 9.62 & 30 \\
10 & 4 & 4 & 2.13 & 1.29 & 1.18 & 0.00 & 10.54 & 30 \\
10 & 4 & 5 & 1.83 & 1.33 & 1.18 & 0.00 & 18.42 & 30 \\
10 & 5 & 3 & 3.10 & 1.60 & 1.33 & 0.21 & 15.95 & 29 \\
10 & 5 & 4 & 2.57 & 1.64 & 1.50 & 0.00 & 5.99 & 30 \\
10 & 5 & 5 & 2.40 & 1.88 & 1.63 & 0.05 & 24.07 & 30 \\
10 & 6 & 3 & 4.10 & 2.15 & 1.77 & 1.30 & 15.40 & 30 \\
10 & 6 & 4 & 3.27 & 2.89 & 2.44 & 0.94 & 17.74 & 29 \\
10 & 6 & 5 & 3.97 & 2.73 & 2.31 & 5.52 & 42.15 & 29 \\
\hline Average: & & 2.08 & 1.38 & 1.18 & 0.37 & 19.56 & \\
\hline & & & & & & & &
\end{tabular}


Table 3: Results of the BRTP linear time and 2BRTP B\&B algorithms for 2BRTP instances, occupancy rate $75 \%$.

\begin{tabular}{rrr|rr|rrrr}
\hline & & \multicolumn{5}{|c|}{ BRTP linear } & \multicolumn{3}{c}{ BRTP B\&B } \\
$S$ & $H$ & $G$ & Rel & E[Rel] & E[Rel $]$ & Time(s) & Gap $(\%)$ & Solved \\
\hline 4 & 4 & 3 & 1.27 & 0.72 & 0.62 & 0.00 & 8.33 & 30 \\
4 & 4 & 4 & 1.53 & 0.80 & 0.71 & 0.00 & 7.10 & 30 \\
4 & 4 & 5 & 1.07 & 0.83 & 0.79 & 0.00 & 8.33 & 30 \\
4 & 5 & 3 & 2.00 & 0.90 & 0.72 & 0.00 & 18.00 & 30 \\
4 & 5 & 4 & 1.57 & 1.10 & 0.93 & 0.00 & 27.76 & 30 \\
4 & 5 & 5 & 1.20 & 1.33 & 1.23 & 0.00 & 14.37 & 30 \\
4 & 6 & 3 & 1.97 & 1.22 & 0.98 & 0.06 & 13.14 & 30 \\
4 & 6 & 4 & 2.03 & 1.57 & 1.33 & 0.00 & 28.87 & 30 \\
4 & 6 & 5 & 1.83 & 1.63 & 1.50 & 0.00 & 15.15 & 30 \\
\hline \hline 6 & 4 & 3 & 1.70 & 0.93 & 0.80 & 0.16 & 11.43 & 30 \\
6 & 4 & 4 & 1.57 & 1.00 & 0.82 & 0.00 & 33.06 & 30 \\
6 & 4 & 5 & 1.20 & 0.97 & 0.87 & 0.00 & 7.02 & 30 \\
6 & 5 & 3 & 1.90 & 0.90 & 0.77 & 0.00 & 9.26 & 30 \\
6 & 5 & 4 & 1.53 & 1.04 & 0.93 & 0.00 & 17.33 & 30 \\
6 & 5 & 5 & 1.87 & 1.28 & 1.17 & 0.00 & 14.12 & 30 \\
6 & 6 & 3 & 2.67 & 1.98 & 1.65 & 0.00 & 32.90 & 30 \\
6 & 6 & 4 & 2.83 & 1.81 & 1.46 & 1.96 & 28.92 & 30 \\
6 & 6 & 5 & 2.00 & 1.95 & 1.73 & 0.01 & 20.08 & 30 \\
\hline \hline 8 & 4 & 3 & 2.20 & 0.98 & 0.90 & 0.00 & 9.77 & 30 \\
8 & 4 & 4 & 1.63 & 1.12 & 1.02 & 0.00 & 14.48 & 30 \\
8 & 4 & 5 & 1.93 & 1.10 & 0.98 & 0.00 & 8.33 & 30 \\
8 & 5 & 3 & 3.20 & 1.70 & 1.40 & 0.00 & 17.09 & 28 \\
8 & 5 & 4 & 2.37 & 1.88 & 1.71 & 0.02 & 16.27 & 30 \\
8 & 5 & 5 & 1.83 & 1.97 & 1.78 & 0.01 & 13.92 & 30 \\
8 & 6 & 3 & 4.97 & 2.50 & 1.98 & 0.12 & 29.94 & 28 \\
8 & 6 & 4 & 3.40 & 2.30 & 1.86 & 0.01 & 36.76 & 28 \\
8 & 6 & 5 & 2.73 & 2.49 & 2.24 & 0.00 & 23.43 & 30 \\
\hline \hline 10 & 4 & 3 & 2.47 & 1.18 & 1.00 & 0.00 & 17.36 & 30 \\
10 & 4 & 4 & 2.90 & 1.56 & 1.36 & 0.01 & 14.30 & 30 \\
10 & 4 & 5 & 2.13 & 1.36 & 1.16 & 0.00 & 21.50 & 30 \\
10 & 5 & 3 & 3.57 & 1.57 & 1.35 & 2.45 & 16.38 & 29 \\
10 & 5 & 4 & 3.30 & 2.03 & 1.62 & 0.45 & 32.40 & 30 \\
10 & 5 & 5 & 3.17 & 1.89 & 1.59 & 0.13 & 23.88 & 29 \\
10 & 6 & 3 & 5.33 & 2.38 & 1.87 & 0.00 & 36.61 & 27 \\
10 & 6 & 4 & 4.00 & 2.98 & 2.56 & 3.97 & 22.17 & 29 \\
10 & 6 & 5 & 3.63 & 2.92 & 2.42 & 0.34 & 29.78 & 29 \\
\hline Average: & & 2.40 & 1.55 & 1.33 & 0.27 & 19.43 & \\
\hline & & & & & & & &
\end{tabular}


Table 4: Results of the BRTP linear time and 2BRTP B\&B algorithms for 2BRTP instances, occupancy rate $80 \%$.

\begin{tabular}{|c|c|c|c|c|c|c|c|c|}
\hline \multirow[b]{2}{*}{$S$} & \multirow[b]{2}{*}{$H$} & \multirow[b]{2}{*}{$G$} & \multicolumn{2}{|c|}{ BRTP linear } & \multicolumn{4}{|c|}{ 2BRTP B\&B } \\
\hline & & & Rel & $\mathrm{E}[\mathrm{Rel}]$ & $\mathrm{E}[\mathrm{Rel}]$ & Time(s) & $\operatorname{Gap}(\%)$ & Solved \\
\hline 4 & 4 & 3 & 1.03 & 0.52 & 0.45 & 0.00 & 0.00 & 30 \\
\hline 4 & 4 & 4 & 0.90 & 1.03 & 0.90 & 0.00 & 10.29 & 30 \\
\hline 4 & 4 & 5 & 1.00 & 0.91 & 0.85 & 0.00 & 10.22 & 30 \\
\hline 4 & 5 & 3 & 2.13 & 1.00 & 0.88 & 0.00 & 8.65 & 30 \\
\hline 4 & 5 & 4 & 1.47 & 1.27 & 1.14 & 0.00 & 7.47 & 30 \\
\hline 4 & 5 & 5 & 1.47 & 1.31 & 1.24 & 0.00 & 17.50 & 30 \\
\hline 4 & 6 & 3 & 1.83 & 1.38 & 1.22 & 0.00 & 15.56 & 30 \\
\hline 4 & 6 & 4 & 2.07 & 1.79 & 1.54 & 0.01 & 29.27 & 30 \\
\hline 4 & 6 & 5 & 1.70 & 1.93 & 1.73 & 0.00 & 17.21 & 30 \\
\hline 6 & 4 & 3 & 2.33 & 1.15 & 0.88 & 0.00 & 30.17 & 30 \\
\hline 6 & 4 & 4 & 1.27 & 1.11 & 0.98 & 0.00 & 7.13 & 30 \\
\hline 6 & 4 & 5 & 1.73 & 1.20 & 1.05 & 0.00 & 23.43 & 30 \\
\hline 6 & 5 & 3 & 2.30 & 1.10 & 0.85 & 0.00 & 33.46 & 30 \\
\hline 6 & 5 & 4 & 2.43 & 1.49 & 1.29 & 0.00 & 22.01 & 29 \\
\hline 6 & 5 & 5 & 2.57 & 2.01 & 1.80 & 0.02 & 16.34 & 30 \\
\hline 6 & 6 & 3 & 3.17 & 1.85 & 1.38 & 0.25 & 42.99 & 30 \\
\hline 6 & 6 & 4 & 3.77 & 2.38 & 1.80 & 10.86 & 58.94 & 29 \\
\hline 6 & 6 & 5 & 2.03 & 2.00 & 1.80 & 0.00 & 17.31 & 30 \\
\hline$\overline{88}$ & $\overline{4}$ & $\overline{3}$ & 1.87 & 1.15 & 0.97 & $\overline{0.00}$ & 27.01 & $\overline{30}$ \\
\hline 8 & 4 & 4 & 1.73 & 1.19 & 1.01 & 0.00 & 27.78 & 30 \\
\hline 8 & 4 & 5 & 1.50 & 1.28 & 1.13 & 0.00 & 22.06 & 30 \\
\hline 8 & 5 & 3 & 3.03 & 1.82 & 1.52 & 0.00 & 22.77 & 30 \\
\hline 8 & 5 & 4 & 2.67 & 2.28 & 1.94 & 0.00 & 22.24 & 30 \\
\hline 8 & 5 & 5 & 1.93 & 1.93 & 1.78 & 0.00 & 15.17 & 30 \\
\hline 8 & 6 & 3 & 3.87 & 2.47 & 1.95 & 2.39 & 23.53 & 30 \\
\hline 8 & 6 & 4 & 2.47 & 2.14 & 1.80 & 0.00 & 43.00 & 30 \\
\hline 8 & 6 & 5 & 3.30 & 2.40 & 2.05 & 0.02 & 33.01 & 29 \\
\hline 10 & $\overline{4}$ & 3 & 2.97 & 1.53 & $\overline{c 1.23}$ & $\overline{00.10}$ & 16.73 & $\overline{30}$ \\
\hline 10 & 4 & 4 & 2.63 & 1.59 & 1.37 & 0.00 & 24.59 & 30 \\
\hline 10 & 4 & 5 & 1.53 & 1.82 & 1.67 & 0.00 & 11.76 & 30 \\
\hline 10 & 5 & 3 & 4.53 & 2.18 & 1.80 & 2.47 & 36.92 & 29 \\
\hline 10 & 5 & 4 & 2.80 & 2.38 & 2.11 & 1.19 & 19.74 & 30 \\
\hline 10 & 5 & 5 & 2.87 & 2.43 & 2.20 & 0.42 & 13.12 & 30 \\
\hline 10 & 6 & 3 & 4.57 & 3.12 & 2.43 & 6.48 & 43.74 & 30 \\
\hline 10 & 6 & 4 & 3.77 & 2.87 & 2.37 & 0.00 & 37.17 & 29 \\
\hline 10 & 6 & 5 & 4.07 & 2.98 & 2.58 & 1.27 & 27.55 & 29 \\
\hline $\mathrm{Av}$ & age: & & 2.43 & 1.75 & 1.49 & 0.71 & 23.22 & \\
\hline
\end{tabular}


Table 5: Results of the BRTP linear time and 2BRTP B\&B algorithms for 2BRTP instances based on Caserta et al. (2012).

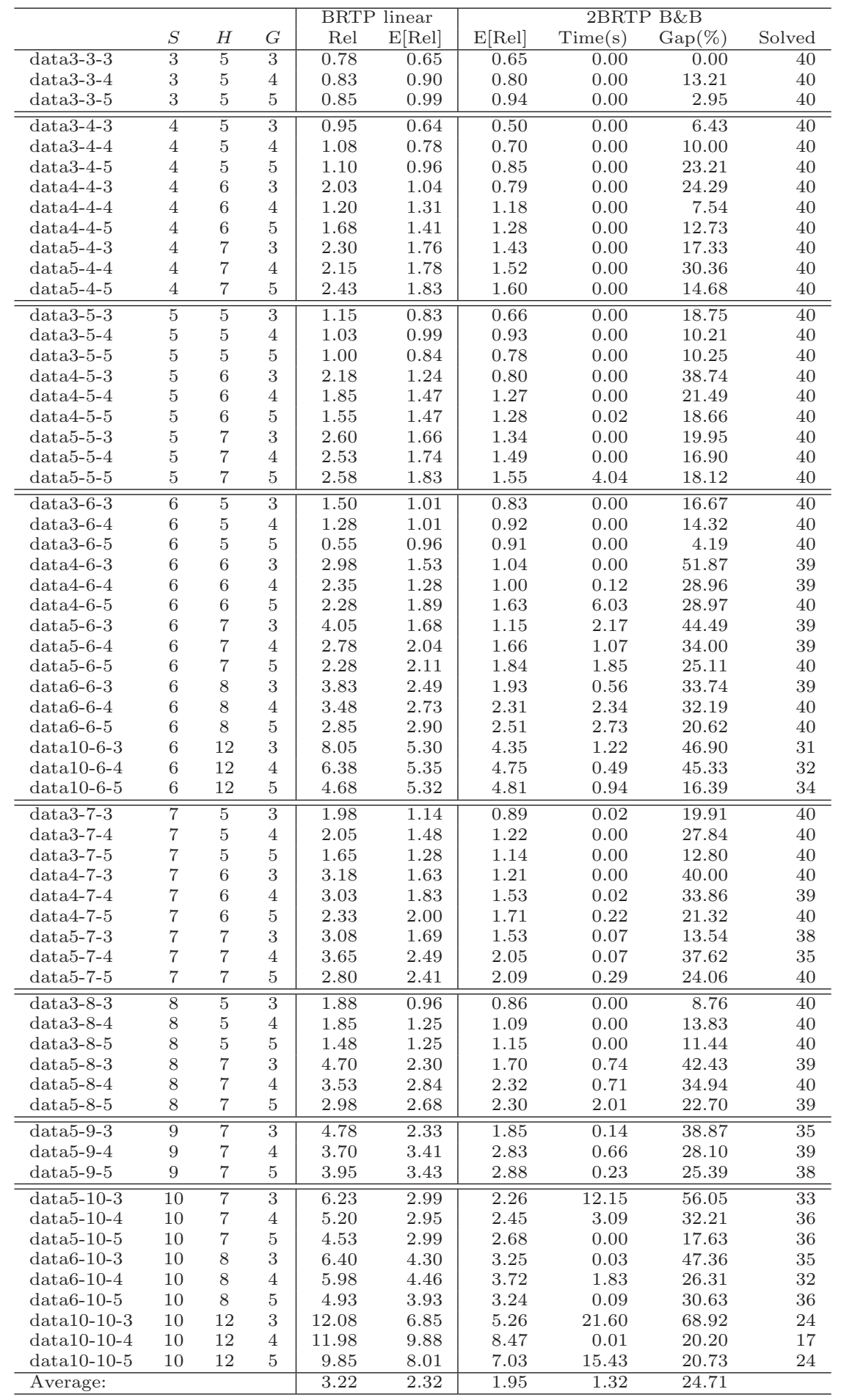


Table 6: Results of the Beam Search on instances with equal retrieval probabilities.

\begin{tabular}{|c|c|c|c|c|c|c|c|}
\hline \multicolumn{8}{|c|}{ Beam Seach - Rule 01} \\
\hline Instance Set & OccRate & $\mathrm{E}[\mathrm{Rel}]$ & Time(s) & BGap(\%) & Solved & Optimal & Less 1s \\
\hline \multirow{3}{*}{ BRTP } & $70 \%$ & 1.18 & 0.10 & 0.06 & 1080 & 1076 & 1078 \\
\hline & $75 \%$ & 1.32 & 0.02 & 0.21 & 1079 & 1065 & 1075 \\
\hline & $80 \%$ & 1.49 & 0.00 & 0.22 & 1080 & 1072 & 1078 \\
\hline Caserta et al. (2012) & $60 \%-85 \%$ & 1.91 & 0.99 & 2.60 & 2467 & 2387 & 2417 \\
\hline \multicolumn{8}{|c|}{ Beam Seach - Rule 02} \\
\hline Instance Set & OccRate & $\mathrm{E}[\mathrm{Rel}]$ & Time(s) & BGap(\%) & Solved & Optimal & Less $1 \mathrm{~s}$ \\
\hline \multirow{3}{*}{ BRTP } & $70 \%$ & 1.18 & 0.00 & 0.01 & 1080 & 1077 & 1080 \\
\hline & $75 \%$ & 1.32 & 0.00 & 0.05 & 1080 & 1067 & 1080 \\
\hline & $80 \%$ & 1.49 & 0.28 & 0.19 & 1080 & 1074 & 1078 \\
\hline Caserta et al. (2012) & $60 \%-85 \%$ & 1.86 & 0.28 & 1.18 & 2513 & 2387 & 2499 \\
\hline \multicolumn{8}{|c|}{ Beam Seach - Rules 01 and 02} \\
\hline Instance Set & OccRate & $\mathrm{E}[\mathrm{Rel}]$ & Time(s) & $\mathrm{BGap}(\%)$ & Solved & Optimal & Less $1 \mathrm{~s}$ \\
\hline \multirow{3}{*}{ BRTP } & $70 \%$ & 1.18 & 0.00 & 0.06 & 1080 & 1076 & 1080 \\
\hline & $75 \%$ & 1.32 & 0.00 & 0.12 & 1080 & 1065 & 1080 \\
\hline & $80 \%$ & 1.49 & 0.00 & 0.22 & 1080 & 1072 & 1080 \\
\hline Caserta et al. (2012) & $60 \%-85 \%$ & 1.86 & 0.01 & 1.07 & 2518 & 2387 & 2516 \\
\hline
\end{tabular}

Table 7: Results of the BRTP linear time and 2BRTP B\&B algorithms for 2BRTP instances, occupancy rate $70 \%$, and unequal retrieval probabilities.

\begin{tabular}{rrr|rr|rrrr}
\hline & & \multicolumn{5}{|c|}{ BRTP linear } & \multicolumn{3}{c}{ 2BRTP B\&B } & B \\
$S$ & $H$ & $G$ & Rel & E[Rel] & E[Rel] & Time(s) & Gap $(\%)$ & Solved \\
\hline 4 & 4 & 3 & 0.47 & 0.60 & 0.58 & 0.00 & 1.79 & 30 \\
4 & 4 & 4 & 0.93 & 0.69 & 0.58 & 0.00 & 17.55 & 30 \\
4 & 4 & 5 & 0.87 & 0.79 & 0.73 & 0.00 & 15.99 & 30 \\
4 & 5 & 3 & 1.40 & 0.76 & 0.58 & 0.00 & 16.77 & 30 \\
4 & 5 & 4 & 1.30 & 1.18 & 0.97 & 0.00 & 39.42 & 30 \\
4 & 5 & 5 & 1.30 & 1.14 & 1.04 & 0.00 & 10.39 & 30 \\
4 & 6 & 3 & 2.03 & 1.31 & 1.13 & 0.00 & 13.90 & 30 \\
4 & 6 & 4 & 1.43 & 1.14 & 1.08 & 0.00 & 3.53 & 30 \\
4 & 6 & 5 & 1.37 & 1.30 & 1.09 & 0.00 & 30.87 & 30 \\
\hline \hline 6 & 4 & 3 & 0.93 & 0.49 & 0.45 & 0.00 & 2.27 & 30 \\
6 & 4 & 4 & 1.23 & 0.91 & 0.79 & 0.00 & 18.72 & 30 \\
6 & 4 & 5 & 0.97 & 0.74 & 0.65 & 0.00 & 14.64 & 30 \\
6 & 5 & 3 & 2.30 & 0.85 & 0.68 & 0.00 & 11.89 & 30 \\
6 & 5 & 4 & 3.13 & 1.24 & 0.96 & 0.08 & 29.09 & 30 \\
6 & 5 & 5 & 1.17 & 1.36 & 1.23 & 0.00 & 17.86 & 30 \\
6 & 6 & 3 & 3.40 & 1.45 & 0.81 & 0.06 & 64.82 & 30 \\
6 & 6 & 4 & 2.90 & 1.70 & 1.43 & 0.01 & 32.70 & 30 \\
6 & 6 & 5 & 1.67 & 1.92 & 1.74 & 0.00 & 20.85 & 30 \\
\hline \hline 8 & 4 & 3 & 1.83 & 0.65 & 0.58 & 0.00 & 4.31 & 30 \\
8 & 4 & 4 & 1.83 & 0.96 & 0.85 & 0.01 & 14.90 & 30 \\
8 & 4 & 5 & 1.20 & 1.02 & 0.94 & 0.00 & 23.73 & 30 \\
8 & 5 & 3 & 2.23 & 1.61 & 1.41 & 0.00 & 8.81 & 30 \\
8 & 5 & 4 & 2.27 & 1.71 & 1.50 & 0.10 & 22.76 & 30 \\
8 & 5 & 5 & 2.13 & 1.68 & 1.45 & 0.02 & 25.23 & 30 \\
8 & 6 & 3 & 3.37 & 2.09 & 1.66 & 4.87 & 31.94 & 30 \\
8 & 6 & 4 & 3.17 & 1.71 & 1.24 & 0.09 & 63.24 & 30 \\
8 & 6 & 5 & 2.40 & 2.01 & 1.78 & 0.01 & 42.63 & 30 \\
\hline \hline 10 & 4 & 3 & 2.20 & 1.36 & 1.24 & 0.00 & 8.38 & 30 \\
10 & 4 & 4 & 2.13 & 1.27 & 1.17 & 0.00 & 9.94 & 30 \\
10 & 4 & 5 & 1.83 & 1.33 & 1.18 & 0.00 & 20.29 & 30 \\
10 & 5 & 3 & 3.10 & 1.62 & 1.32 & 0.20 & 20.12 & 29 \\
10 & 5 & 4 & 2.57 & 1.62 & 1.48 & 0.00 & 5.41 & 30 \\
10 & 5 & 5 & 2.40 & 1.97 & 1.72 & 0.17 & 23.40 & 30 \\
10 & 6 & 3 & 4.10 & 2.13 & 1.75 & 1.91 & 15.41 & 30 \\
10 & 6 & 4 & 3.27 & 2.91 & 2.47 & 1.07 & 18.52 & 29 \\
10 & 6 & 5 & 3.97 & 2.60 & 2.21 & 0.09 & 52.61 & 28 \\
\hline Average: & & 2.08 & 1.38 & 1.18 & 0.24 & 21.52 & \\
\hline & & & & & & & &
\end{tabular}


Table 8: Results of the BRTP linear time and 2BRTP B\&B algorithms for 2BRTP instances, occupancy rate $75 \%$, and unequal retrieval probabilities.

\begin{tabular}{|c|c|c|c|c|c|c|c|c|}
\hline \multirow[b]{2}{*}{$S$} & \multirow[b]{2}{*}{$H$} & \multirow[b]{2}{*}{$G$} & \multicolumn{2}{|c|}{ BRTP linear } & \multicolumn{4}{|c|}{ 2BRTP B\&B } \\
\hline & & & Rel & $\mathrm{E}[\mathrm{Rel}]$ & $\mathrm{E}[\mathrm{Rel}]$ & Time(s) & $\operatorname{Gap}(\%)$ & Solved \\
\hline 4 & 4 & 3 & 1.27 & 0.71 & 0.61 & 0.00 & 10.49 & 30 \\
\hline 4 & 4 & 4 & 1.53 & 0.80 & 0.70 & 0.00 & 8.13 & 30 \\
\hline 4 & 4 & 5 & 1.07 & 0.86 & 0.80 & 0.00 & 11.82 & 30 \\
\hline 4 & 5 & 3 & 2.00 & 0.91 & 0.71 & 0.00 & 22.18 & 30 \\
\hline 4 & 5 & 4 & 1.57 & 1.12 & 0.94 & 0.00 & 30.67 & 30 \\
\hline 4 & 5 & 5 & 1.20 & 1.39 & 1.27 & 0.00 & 13.97 & 30 \\
\hline 4 & 6 & 3 & 1.97 & 1.13 & 0.90 & 0.07 & 11.62 & 30 \\
\hline 4 & 6 & 4 & 2.03 & 1.49 & 1.26 & 0.00 & 30.50 & 30 \\
\hline 4 & 6 & 5 & 1.83 & 1.58 & 1.44 & 0.00 & 24.94 & 30 \\
\hline 6 & 4 & 3 & 1.70 & 0.92 & 0.77 & $\overline{0.19}$ & 15.39 & 30 \\
\hline 6 & 4 & 4 & 1.57 & 0.99 & 0.80 & 0.00 & 34.06 & 30 \\
\hline 6 & 4 & 5 & 1.20 & 0.96 & 0.84 & 0.00 & 10.86 & 30 \\
\hline 6 & 5 & 3 & 1.90 & 0.95 & 0.84 & 0.00 & 9.22 & 30 \\
\hline 6 & 5 & 4 & 1.53 & 1.06 & 0.92 & 0.00 & 24.24 & 30 \\
\hline 6 & 5 & 5 & 1.87 & 1.36 & 1.23 & 0.00 & 16.47 & 30 \\
\hline 6 & 6 & 3 & 2.67 & 2.05 & 1.75 & 0.00 & 28.16 & 30 \\
\hline 6 & 6 & 4 & 2.83 & 1.83 & 1.44 & 2.28 & 32.15 & 30 \\
\hline 6 & 6 & 5 & 2.00 & 2.01 & 1.76 & 0.02 & 19.66 & 30 \\
\hline$\overline{88}$ & $\overline{\overline{4}}$ & $\overline{3}$ & 2.20 & $\overline{~ 1.02}$ & $\overline{0.93}$ & $\overline{\overline{0.00}}$ & 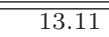 & $\overline{30}$ \\
\hline 8 & 4 & 4 & 1.63 & 1.12 & 1.02 & 0.00 & 13.37 & 30 \\
\hline 8 & 4 & 5 & 1.93 & 1.10 & 0.98 & 0.00 & 9.23 & 30 \\
\hline 8 & 5 & 3 & 3.20 & 1.74 & 1.44 & 0.00 & 18.21 & 28 \\
\hline 8 & 5 & 4 & 2.37 & 1.96 & 1.79 & 0.00 & 18.69 & 30 \\
\hline 8 & 5 & 5 & 1.83 & 1.97 & 1.79 & 0.01 & 13.32 & 30 \\
\hline 8 & 6 & 3 & 4.97 & 2.38 & 1.85 & 0.37 & 35.20 & 27 \\
\hline 8 & 6 & 4 & 3.40 & 2.32 & 1.86 & 0.01 & 37.34 & 28 \\
\hline 8 & 6 & 5 & 2.73 & 2.50 & 2.22 & 0.00 & 23.64 & 30 \\
\hline 10 & $\overline{4}$ & 3 & 2.47 & 1.25 & 1.05 & $\overline{00.00}$ & 21.50 & $\overline{30}$ \\
\hline 10 & 4 & 4 & 2.90 & 1.59 & 1.40 & 0.03 & 14.56 & 30 \\
\hline 10 & 4 & 5 & 2.13 & 1.37 & 1.17 & 0.00 & 23.28 & 30 \\
\hline 10 & 5 & 3 & 3.57 & 1.62 & 1.36 & 2.66 & 20.85 & 29 \\
\hline 10 & 5 & 4 & 3.30 & 2.03 & 1.60 & 0.36 & 37.54 & 30 \\
\hline 10 & 5 & 5 & 3.17 & 1.80 & 1.50 & 0.24 & 30.60 & 29 \\
\hline 10 & 6 & 3 & 5.33 & 2.37 & 1.90 & 0.00 & 37.72 & 27 \\
\hline 10 & 6 & 4 & 4.00 & 2.93 & 2.48 & 0.01 & 21.07 & 28 \\
\hline 10 & 6 & 5 & 3.63 & 2.82 & 2.30 & 0.55 & 34.80 & 29 \\
\hline \multicolumn{3}{|c|}{ Average: } & 2.40 & 1.56 & 1.32 & 0.19 & 21.63 & \\
\hline
\end{tabular}


Table 9: Results of the BRTP linear time and 2BRTP B\&B algorithms for 2BRTP instances, occupancy rate $80 \%$, and unequal retrieval probabilities.

\begin{tabular}{|c|c|c|c|c|c|c|c|c|}
\hline \multirow[b]{2}{*}{$S$} & \multirow[b]{2}{*}{$H$} & \multirow[b]{2}{*}{$G$} & \multicolumn{2}{|c|}{ BRTP linear } & \multicolumn{4}{|c|}{ 2BRTP B\&B } \\
\hline & & & Rel & $\mathrm{E}[\mathrm{Rel}]$ & $\mathrm{E}[\mathrm{Rel}]$ & Time(s) & $\operatorname{Gap}(\%)$ & Solved \\
\hline 4 & 4 & 3 & 1.03 & 0.52 & 0.46 & 0.00 & 0.00 & 30 \\
\hline 4 & 4 & 4 & 0.90 & 1.03 & 0.89 & 0.00 & 10.45 & 30 \\
\hline 4 & 4 & 5 & 1.00 & 0.86 & 0.78 & 0.00 & 25.60 & 30 \\
\hline 4 & 5 & 3 & 2.13 & 1.12 & 0.99 & 0.00 & 14.85 & 30 \\
\hline 4 & 5 & 4 & 1.47 & 1.27 & 1.15 & 0.00 & 7.83 & 30 \\
\hline 4 & 5 & 5 & 1.47 & 1.29 & 1.21 & 0.00 & 40.67 & 30 \\
\hline 4 & 6 & 3 & 1.83 & 1.42 & 1.26 & 0.00 & 13.36 & 30 \\
\hline 4 & 6 & 4 & 2.07 & 1.76 & 1.50 & 0.01 & 38.67 & 30 \\
\hline 4 & 6 & 5 & 1.70 & 1.92 & 1.73 & 0.00 & 16.00 & 30 \\
\hline 6 & 4 & 3 & 2.33 & 1.19 & 0.91 & 0.00 & 29.19 & 30 \\
\hline 6 & 4 & 4 & 1.27 & 1.12 & 0.97 & 0.00 & 9.70 & 30 \\
\hline 6 & 4 & 5 & 1.73 & 1.25 & 1.07 & 0.00 & 31.60 & 30 \\
\hline 6 & 5 & 3 & 2.30 & 1.17 & 0.91 & 0.00 & 39.45 & 30 \\
\hline 6 & 5 & 4 & 2.43 & 1.47 & 1.25 & 0.00 & 23.58 & 29 \\
\hline 6 & 5 & 5 & 2.57 & 1.97 & 1.77 & 0.02 & 19.23 & 30 \\
\hline 6 & 6 & 3 & 3.17 & 1.69 & 1.27 & 0.26 & 35.03 & 30 \\
\hline 6 & 6 & 4 & 3.77 & 2.42 & 1.82 & 1.24 & 61.73 & 28 \\
\hline 6 & 6 & 5 & 2.03 & 2.11 & 1.85 & 0.00 & 20.08 & 30 \\
\hline$\overline{88}$ & $\overline{4}$ & $\overline{3}$ & 1.87 & 1.18 & 0.98 & $\overline{0.00}$ & 27.54 & $\overline{30}$ \\
\hline 8 & 4 & 4 & 1.73 & 1.21 & 1.05 & 0.00 & 25.63 & 30 \\
\hline 8 & 4 & 5 & 1.50 & 1.28 & 1.11 & 0.00 & 28.92 & 30 \\
\hline 8 & 5 & 3 & 3.03 & 1.79 & 1.48 & 0.00 & 27.70 & 30 \\
\hline 8 & 5 & 4 & 2.67 & 2.33 & 2.00 & 0.00 & 19.68 & 30 \\
\hline 8 & 5 & 5 & 1.93 & 1.96 & 1.80 & 0.00 & 18.13 & 30 \\
\hline 8 & 6 & 3 & 3.87 & 2.41 & 1.88 & 4.42 & 26.87 & 30 \\
\hline 8 & 6 & 4 & 2.47 & 2.16 & 1.78 & 0.00 & 43.75 & 30 \\
\hline 8 & 6 & 5 & 3.30 & 2.43 & 2.07 & 0.05 & 35.14 & 29 \\
\hline 10 & $\overline{4}$ & 3 & 2.97 & 1.64 & 21.30 & $\overline{00.10}$ & 217.30 & $\overline{30}$ \\
\hline 10 & 4 & 4 & 2.63 & 1.62 & 1.38 & 0.00 & 26.02 & 30 \\
\hline 10 & 4 & 5 & 1.53 & 1.88 & 1.73 & 0.00 & 12.42 & 30 \\
\hline 10 & 5 & 3 & 4.53 & 2.36 & 1.95 & 2.84 & 35.98 & 29 \\
\hline 10 & 5 & 4 & 2.80 & 2.36 & 2.09 & 1.33 & 19.41 & 30 \\
\hline 10 & 5 & 5 & 2.87 & 2.40 & 2.15 & 0.11 & 16.47 & 30 \\
\hline 10 & 6 & 3 & 4.57 & 3.16 & 2.46 & 6.92 & 57.97 & 30 \\
\hline 10 & 6 & 4 & 3.77 & 2.93 & 2.40 & 0.65 & 35.75 & 29 \\
\hline 10 & 6 & 5 & 4.07 & 2.91 & 2.51 & 1.81 & 26.26 & 29 \\
\hline $\mathrm{Av}$ & age: & & 2.43 & 1.77 & 1.50 & 0.55 & 26.05 & \\
\hline
\end{tabular}


Table 10: Results of the BRTP linear time and 2BRTP B\&B algorithms for 2BRTP instances based on Caserta et al. (2012), and unequal retrieval probabilities.

\begin{tabular}{|c|c|c|c|c|c|c|c|c|c|}
\hline \multirow[b]{2}{*}{ Instance } & \multirow[b]{2}{*}{$S$} & \multirow[b]{2}{*}{$H$} & \multirow[b]{2}{*}{$G$} & \multicolumn{2}{|c|}{ BRTP linear } & \multicolumn{4}{|c|}{ 2BRTP B\&B } \\
\hline & & & & Rel & $\mathrm{E}[$ Rel $]$ & $\mathrm{E}[\mathrm{Rel}]$ & Times) & Gap (\%) & Solved \\
\hline data3-3-3 & 3 & 5 & 3 & 0.78 & 0.73 & 0.73 & 0.00 & 0.00 & 40 \\
\hline data3-3-4 & 3 & 5 & 4 & 0.83 & 0.89 & 0.79 & 0.00 & 13.13 & 40 \\
\hline data3-3-5 & 3 & 5 & 5 & 0.85 & 0.95 & 0.91 & 0.00 & 2.92 & 40 \\
\hline data3-4-3 & 4 & 5 & 3 & 0.95 & 0.62 & 0.48 & 0.00 & 6.83 & $\overline{40}$ \\
\hline data3-4-4 & 4 & 5 & 4 & 1.08 & 0.77 & 0.68 & 0.00 & 12.00 & 40 \\
\hline data3 $3-4-5$ & 4 & 5 & 5 & 1.10 & 0.94 & 0.83 & 0.00 & 33.55 & 40 \\
\hline data $4-4-3$ & 4 & 6 & 3 & 2.03 & 1.07 & 0.81 & 0.00 & 31.93 & 40 \\
\hline data $4-4-4$ & 4 & 6 & 4 & 1.20 & 1.26 & 1.13 & 0.00 & 10.49 & 40 \\
\hline data4-4-5 & 4 & 6 & 5 & 1.68 & 1.37 & 1.21 & 0.00 & 21.94 & 40 \\
\hline data5-4-3 & 4 & 7 & 3 & 2.30 & 1.70 & 1.39 & 0.00 & 16.71 & 40 \\
\hline data5-4-4 & 4 & 7 & 4 & 2.15 & 1.78 & 1.49 & 0.00 & 31.63 & 40 \\
\hline data5-4-5 & 4 & 7 & 5 & 2.43 & 1.77 & 1.51 & 0.00 & 20.08 & 40 \\
\hline data3-5-3 & $\overline{5}$ & $\overline{5}$ & $\overline{3}$ & 1.15 & $\overline{0.82}$ & $\overline{0.66}$ & 0.00 & 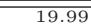 & $\overline{40}$ \\
\hline data3-5-4 & 5 & 5 & 4 & 1.03 & 0.96 & 0.89 & 0.00 & 10.50 & 40 \\
\hline data3-5-5 & 5 & 5 & 5 & 1.00 & 0.87 & 0.78 & 0.00 & 12.71 & 40 \\
\hline data $4-5-3$ & 5 & 6 & 3 & 2.18 & 1.09 & 0.69 & 0.00 & 31.91 & 40 \\
\hline data4-5-4 & 5 & 6 & 4 & 1.85 & 1.47 & 1.25 & 0.00 & 23.89 & 40 \\
\hline data4-5-5 & 5 & 6 & 5 & 1.55 & 1.46 & 1.24 & 0.02 & 25.53 & 40 \\
\hline data5-5-3 & 5 & 7 & 3 & 2.60 & 1.60 & 1.25 & 0.00 & 24.91 & 40 \\
\hline data5-5-4 & 5 & 7 & 4 & 2.53 & 1.71 & 1.45 & 0.00 & 17.53 & 40 \\
\hline data5-5-5 & 5 & 7 & 5 & 2.58 & 1.93 & 1.58 & 5.30 & 22.48 & 40 \\
\hline data3-6-3 & $\overline{c 6}$ & $\overline{5}$ & $\overline{3}$ & 1.50 & 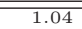 & 0.85 & 0.00 & 15.35 & 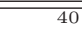 \\
\hline data3-6-4 & 6 & 5 & 4 & 1.28 & 1.00 & 0.91 & 0.00 & 15.89 & 40 \\
\hline data3-6-5 & 6 & 5 & 5 & 0.55 & 0.91 & 0.87 & 0.00 & 3.92 & 40 \\
\hline data $4-6-3$ & 6 & 6 & 3 & 2.98 & 1.51 & 1.05 & 0.00 & 51.46 & 39 \\
\hline data $4-6-4$ & 6 & 6 & 4 & 2.35 & 1.28 & 0.99 & 0.20 & 34.62 & 39 \\
\hline data4-6-5 & 6 & 6 & 5 & 2.28 & 1.85 & 1.57 & 0.46 & 28.36 & 39 \\
\hline data5-6-3 & 6 & 7 & 3 & 4.05 & 1.72 & 1.17 & 3.59 & 46.60 & 39 \\
\hline data5-6-4 & 6 & 7 & 4 & 2.78 & 1.97 & 1.60 & 1.17 & 38.73 & 39 \\
\hline data5-6-5 & 6 & 7 & 5 & 2.28 & 2.17 & 1.86 & 3.75 & 27.45 & 40 \\
\hline data6-6-3 & 6 & 8 & 3 & 3.83 & 2.53 & 2.00 & 1.90 & 35.88 & 39 \\
\hline data6-6-4 & 6 & 8 & 4 & 3.48 & 2.70 & 2.27 & 4.13 & 34.38 & 40 \\
\hline data6-6-5 & 6 & 8 & 5 & 2.85 & 2.98 & 2.55 & 2.62 & 25.16 & 40 \\
\hline data10-6-3 & 6 & 12 & 3 & 8.05 & 5.35 & 4.42 & 1.95 & 36.61 & 31 \\
\hline data10-6-4 & 6 & 12 & 4 & 6.38 & 5.37 & 4.72 & 0.62 & 46.48 & 32 \\
\hline data10-6-5 & 6 & 12 & 5 & 4.68 & 5.11 & 4.53 & 0.23 & 20.93 & 34 \\
\hline data3-7-3 & $\overline{77}$ & $\bar{~} 5$ & 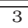 & 1.98 & 1.10 & 0.87 & 0.02 & 22.58 & $\overline{440}$ \\
\hline data3-7-4 & 7 & 5 & 4 & 2.05 & 1.45 & 1.18 & 0.00 & 29.31 & 40 \\
\hline data3-7-5 & 7 & 5 & 5 & 1.65 & 1.28 & 1.13 & 0.00 & 13.81 & 40 \\
\hline data $4-7-3$ & 7 & 6 & 3 & 3.18 & 1.60 & 1.21 & 0.00 & 40.85 & 40 \\
\hline data $4-7-4$ & 7 & 6 & 4 & 3.03 & 1.87 & 1.55 & 0.03 & 35.24 & 39 \\
\hline data4-7-5 & 7 & 6 & 5 & 2.33 & 2.05 & 1.72 & 0.41 & 31.65 & 40 \\
\hline data5-7-3 & 7 & 7 & 3 & 3.08 & 1.75 & 1.60 & 0.08 & 12.96 & 38 \\
\hline data5-7-4 & 7 & 7 & 4 & 3.65 & 2.49 & 2.05 & 0.07 & 42.91 & 35 \\
\hline data5-7-5 & 7 & 7 & 5 & 2.80 & 2.44 & 2.08 & 1.61 & 25.87 & 40 \\
\hline data3-8-3 & 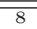 & $\overline{5}$ & 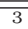 & 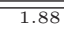 & 0.93 & $\overline{0.83}$ & $\overline{0.00}$ & 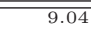 & $\overline{\overline{40}}$ \\
\hline data3-8-4 & 8 & 5 & 4 & 1.85 & 1.25 & 1.08 & 0.00 & 14.94 & 40 \\
\hline data3-8-5 & 8 & 5 & 5 & 1.48 & 1.31 & 1.21 & 0.00 & 11.46 & 40 \\
\hline data5-8-3 & 8 & 7 & 3 & 4.70 & 2.32 & 1.68 & 0.73 & 41.88 & 39 \\
\hline data5-8-4 & 8 & 7 & 4 & 3.53 & 2.76 & 2.25 & 3.41 & 37.77 & 40 \\
\hline data5-8-5 & 8 & 7 & 5 & 2.98 & 2.67 & 2.25 & 2.28 & 26.97 & 39 \\
\hline data5-9-3 & 9 & 7 & 3 & 4.78 & 2.27 & 1.83 & 0.22 & 37.01 & 35 \\
\hline data5-9-4 & 9 & 7 & 4 & 3.70 & 3.40 & 2.82 & 1.27 & 28.92 & 39 \\
\hline data5-9-5 & 9 & 7 & 5 & 3.95 & 3.41 & 2.77 & 0.37 & 31.02 & 38 \\
\hline data5-10-3 & 10 & 7 & 3 & 6.23 & 2.84 & 2.21 & 15.03 & 62.35 & 33 \\
\hline data5-10-4 & 10 & 7 & 4 & 5.20 & 3.07 & 2.55 & 2.69 & 31.68 & 36 \\
\hline data5-10-5 & 10 & 7 & 5 & 4.53 & 3.00 & 2.63 & 0.49 & 20.38 & 36 \\
\hline data6-10-3 & 10 & 8 & 3 & 6.40 & 4.42 & 3.36 & 0.14 & 43.97 & 34 \\
\hline data6-10-4 & 10 & 8 & 4 & 5.98 & 4.45 & 3.66 & 0.39 & 30.15 & 32 \\
\hline data6-10-5 & 10 & 8 & 5 & 4.93 & 4.12 & 3.46 & 0.48 & 23.47 & 36 \\
\hline data10-10-3 & 10 & 12 & 3 & 12.08 & 6.89 & 5.56 & 15.35 & 51.42 & 21 \\
\hline data10-10-4 & 10 & 12 & 4 & 11.98 & 9.85 & 8.43 & 2.49 & 21.06 & 16 \\
\hline data10-10-5 & 10 & 12 & 5 & 9.85 & 7.93 & 6.93 & 4.02 & 21.45 & 22 \\
\hline Average: & & & & 3.22 & 2.32 & 1.94 & 1.23 & 26.23 & \\
\hline
\end{tabular}


Table 11: Beam Search results for unequal retrieval probabilities.

\begin{tabular}{|c|c|c|c|c|c|c|c|}
\hline \multicolumn{8}{|c|}{ Beam Seach - Rule 01} \\
\hline Instance Set & OccRate & E[Rel] & Time(s) & BGap(\%) & Solved & Optimal & Less $1 \mathrm{~s}$ \\
\hline \multirow{3}{*}{ BRTP } & $70 \%$ & 1.18 & 0.01 & 0.06 & 1079 & 1075 & 1078 \\
\hline & $75 \%$ & 1.31 & 0.04 & 0.18 & 1079 & 1061 & 1075 \\
\hline & $80 \%$ & 1.49 & 0.01 & 0.27 & 1080 & 1070 & 1078 \\
\hline Caserta et al. (2012) & $60 \%-85 \%$ & 1.90 & 1.25 & 2.88 & 2460 & 2377 & 2414 \\
\hline \multicolumn{8}{|c|}{ Beam Seach - Rule 02} \\
\hline Instance Set & OccRate & $\mathrm{E}[\mathrm{Rel}]$ & Time(s) & BGap(\%) & Solved & Optimal & Less 1s \\
\hline \multirow{3}{*}{ BRTP } & $70 \%$ & 1.18 & 0.00 & 0.01 & 1080 & 1076 & 1080 \\
\hline & $75 \%$ & 1.31 & 0.01 & 0.04 & 1080 & 1065 & 1079 \\
\hline & $80 \%$ & 1.49 & 0.00 & 0.21 & 1078 & 1073 & 1078 \\
\hline Caserta et al. (2012) & $60 \%-85 \%$ & 1.85 & 0.37 & 1.38 & 2510 & 2379 & 2493 \\
\hline \multicolumn{8}{|c|}{ Beam Seach - Rules 01 and 02} \\
\hline Instance Set & OccRate & $\mathrm{E}[\mathrm{Rel}]$ & Time(s) & BGap(\%) & Solved & Optimal & Less $1 \mathrm{~s}$ \\
\hline \multirow{3}{*}{ BRTP } & $70 \%$ & 1.18 & 0.00 & 0.06 & 1080 & 1075 & 1080 \\
\hline & $75 \%$ & 1.31 & 0.00 & 0.09 & 1080 & 1061 & 1080 \\
\hline & $80 \%$ & 1.49 & 0.00 & 0.27 & 1080 & 1070 & 1080 \\
\hline Caserta et al. (2012) & $60 \%-85 \%$ & 1.85 & 0.01 & 0.99 & 2517 & 2377 & 2513 \\
\hline
\end{tabular}

Table 12: Results of the Tanaka and Takii (2016) BRP B\&B algorithm and the BRTP B\&B algorithm on BRTP instances.

\begin{tabular}{rrr|rrr|rrr}
\hline \multicolumn{2}{|c|}{ Instance Set } & \multicolumn{2}{|c|}{ Literature B\&B algorithm } & \multicolumn{3}{c}{ Our B\&B algorithm } \\
$G$ & $S$ & $H$ & Rel & Time(s) & Solved & Rel & Time(s) & Solved \\
\hline 2 & 10 & 10 & 46.176 & 0.0062 & 1000 & 46.176 & 0.0002 & 1000 \\
3 & 10 & 10 & 35.769 & 0.0024 & 1000 & 35.769 & 0.0000 & 1000 \\
2 & 10 & 15 & 81.921 & 6.2845 & 987 & 81.870 & 1.2755 & 998 \\
3 & 10 & 15 & 68.415 & 2.6506 & 983 & 68.395 & 0.1157 & 998 \\
\hline
\end{tabular}

